\title{
A Critical Review of Computational Methods and Their Application in Industrial Fan Design
}

\author{
Alessandro Corsini, ${ }^{1}$ Giovanni Delibra, ${ }^{1}$ and Anthony G. Sheard ${ }^{2}$ \\ ${ }^{1}$ Dipartimento di Ingegneria Meccanica e Aerospaziale, Sapienza University of Rome, Via Eudossiana 18, 00184 Rome, Italy \\ ${ }^{2}$ Fläkt Woods Limited, Axial Way, Colchester CO4 5ZD, UK \\ Correspondence should be addressed to Anthony G. Sheard; geoff.sheard@flaktwoods.com
}

Received 13 August 2013; Accepted 23 September 2013

Academic Editors: O. Allix, S. W. Chang, and R.-J. Yang

Copyright (c) 2013 Alessandro Corsini et al. This is an open access article distributed under the Creative Commons Attribution License, which permits unrestricted use, distribution, and reproduction in any medium, provided the original work is properly cited.

\begin{abstract}
Members of the aerospace fan community have systematically developed computational methods over the last five decades. The complexity of the developed methods and the difficulty associated with their practical application ensured that, although commercial computational codes date back to the 1980s, they were not fully exploited by industrial fan designers until the beginning of the 2000s. The application of commercial codes proved to be problematic as, unlike aerospace fans, industrial fans include electrical motors and other components from which the flow will invariably separate. Consequently, industrial fan designers found the application of commercial codes challenging. The decade from 2000 to 2010 was focused on developing techniques that would facilitate converged solutions that predicted the fans' performance characteristics over the stable part of their operating range with reasonable accuracy, using a practical computational effort. In this paper, we focus on elucidating aspects of the flow physics that one cannot easily study in a laboratory environment, discussing the challenges involved and the relative merits of the available modelling techniques. The paper ends with a discussion of the practical problems associated with the use of commercial codes in a development environment and finally the legislation that is driving the need for aerospace style computation methods.
\end{abstract}

\section{Introduction}

Industrial fan designers have historically relied on empirical design methodologies based upon an Euler analysis of velocity triangles [1], empirical correlations [2], experimental fluid dynamics [3], and fan noise measurements [4, 5]. Engineers have developed these empirical design methodologies over many decades, with each industrial fan manufacturer developing its own proprietary empirical correlations that aid in applying the basic methodology in specific applications. Unlike aerospace fan designers, industrial fan designers have to apply their design methodologies into a wide range of different applications. At one extreme are the cooling fans required for electronic equipment that can be no more than a few centimetres in diameter [6], and at the other extreme are fans absorbing up to $25 \mathrm{MW}$ in induced draft power plant applications [7]. It is this breadth of application that has resulted in the different empirical approaches adopted by competing industrial fan manufacturers, each treating their proprietary empirical approaches as a source of competitive advantage.

The historic view that the empirical approaches to industrial fan design constitute a form of competitive advantage has resulted in a lack of cooperation and collaboration between industrial fan manufacturers. In contrast, aerospace fan designers have cooperated and collaborated, with the result that the available computational methods have progressed steadily over the last five decades. A result has been steadily improving aerospace fan efficiencies, with the drive for improved fan efficiency originating from efficiency as a source of competitive advantage. In the industrial fan community low cost has historically been the primary source of competitive advantage. However, this focus on lowest cost has recently changed because of new regulations in the European Community and planned regulation in the USA.

Within the European Union (EU), Commission Regulation number 327/2011 became legally binding on 1 January 2013, setting minimum fan and motor efficiency grades 
(FMEGs) for commercial and industrial fans [8]. For applicable fans the 2013 minimum fan and motor efficiency grades have resulted in approximately 33 percent of those fans being sold before 1 January 2013, now illegal within Europe [9]. Moreover, these minimum fan and motor efficiency grades will become more stringent on 1 January 2015 [9].

In the USA, the US Department of Energy has been monitoring activity within the European Union. On 1 February 2013 the US federal government published a framework document in the Federal Register, outlining the approach to fan efficiency regulation within the USA [10]. The framework reflected a desire to be consistent with many elements of the European approach in EU Commission Regulation number 327/2011 [10].

With both Europe and the USA now regulating or declaring intent to do so, it is likely that Asian countries will introduce regulations setting minimum industrial fan or fan and motor efficiencies. Currently, Malaysia, Korea, and Taiwan have considered adopting fan efficiency requirements based on the Air Movement and Control Association (AMCA) Standard 205 Energy Efficiency Classification for Fans, as a mandatory requirement for government and private-sector projects [11].

Given today's regulatory environment it is reasonable to assume that in all global regions, minimum fan or fan and motors efficiencies will become mandatory and then increase over time. As a direct result, the pressure on industrial fan designers will increase to both develop fans with high peak efficiency and specify them such that they operate closer to their peak efficiency point when installed. In response to this pressure, industrial fan designers have started to adapt aerospace fan design methodologies for application in the industrial fan design process [12], with a particular emphasis on the use of computational fluid dynamics (CFD). Unlike aerospace fan designers who have the resources to develop their own CFD codes, industrial fan designers must rely on commercial CFD codes. The industrial fan community is fragmented, with no individual fan manufacturers having the resources to develop in-house CFD codes.

Commercial CFD codes first became available in the early 1980s. However, modelling the physical flow equations involved significantly simplifying assumptions. The mesh generation techniques were rudimentary, and the available hardware to run the codes lacked computational power with the result that meshes were inevitably coarse. Over the last three decades, engineers have systematically addressed these issues, and today commercially available CFD codes model the flow equations accurately. Engineers can construct wellconditioned meshes and the available hardware is adequate to achieve mesh-independent results. Consequently, it is now possible to predict an industrial fan's performance characteristics using commercially available CFD codes with reasonable accuracy.

In this paper, we first provide an overview of the computational methods utilised in the industrial fan design process. We start with a description of Reynolds-Averaged NavierStokes (RANS) turbulence modelling. We then move on to consider how one may apply the RANS technique in practice, followed by a consideration of the application of unsteady
RANS, hybrid large eddy simulation (LES)/RANS, and Large Eddy Simulation as part of the industrial fan design process. We conclude with a consideration of the potential of opensource CFD codes.

\section{RANS Turbulence Modelling}

An issue when considering using CFD in any turbomachinery application is that, even in the most favourable cases, Reynolds numbers are high resulting in complex flowfields. A direct numerical simulation (DNS) of the NavierStokes equations would require fine computational grids and therefore a computational effort beyond that available within the industrial fan community. Consequently, the only viable approach available to industrial fan designers when attempting to solve the Navier-Stokes equations is RANS. A key aspect of a RANS simulation is using turbulence models that constitute a range of simplifying computational approaches for modelling aspects of the blade-to-blade flowfield fluid dynamics [13-17]. These simplifying assumptions allow Reynolds-averaging to reduce the computational effort that occurs with a direct numerical simulation of the NavierStokes equations and therefore facilitate simulation of high Reynolds number flows.

Reynolds-Averaged Navier-Stokes equations refers to a process of Reynolds-averaging the quantities within the equations. We express the generic $\varphi$ quantity as the sum of its mean value $\bar{\varphi}$ and the fluctuating value $\varphi^{\prime}$ :

$$
\varphi=\bar{\varphi}+\varphi^{\prime} .
$$

Successive averaging of the equations themselves results in the creation of a series of additional unknowns called Reynolds stresses and in the case of heat transfer, Reynolds fluxes. Solving the RANS equations requires a closed system of equations modelling these additional unknowns.

Moving from a direct numerical simulation to a RANS simulation reduces the required computational effort for a converged solution to a level practical within the industrial fan community. However, the tradeoff is a need to develop methods of modelling the turbulent flow's underlying physics. This requirement is responsible for the significance of turbulence models in RANS codes. The appropriate choice of turbulence model for the application has a primary influence on the accuracy of the fan blade-to-blade flow-field's resulting prediction.

2.1. RANS Models. For incompressible flows (without heat transfer), the RANS equations read

$$
\begin{gathered}
\frac{\partial U_{k}}{\partial x_{k}}=0 \\
\frac{\partial U_{i}}{\partial t}+U_{k} \frac{\partial U_{i}}{\partial x_{k}}=-\frac{1}{\rho} \frac{\partial P}{\partial x_{i}}+v \frac{\partial U_{i}}{\partial x_{j} \partial x_{j}}-\frac{\partial}{\partial x_{k}}\left(\overline{u_{i}^{\prime} u_{k}^{\prime}}\right) .
\end{gathered}
$$

In (3) the term in parentheses is the additional unknown Reynolds stress that requires modelling. Most models assume 


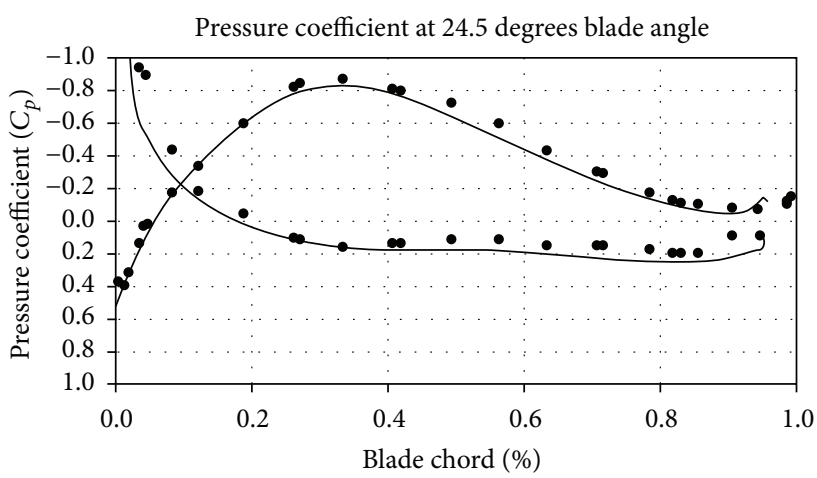

(a)

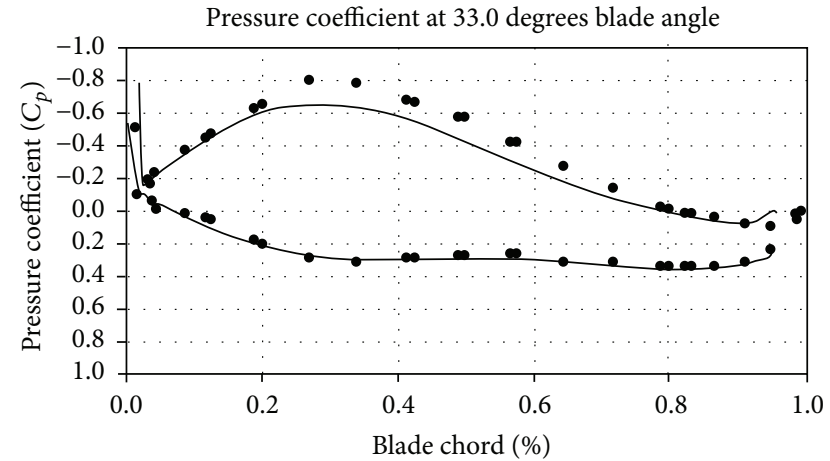

(b)

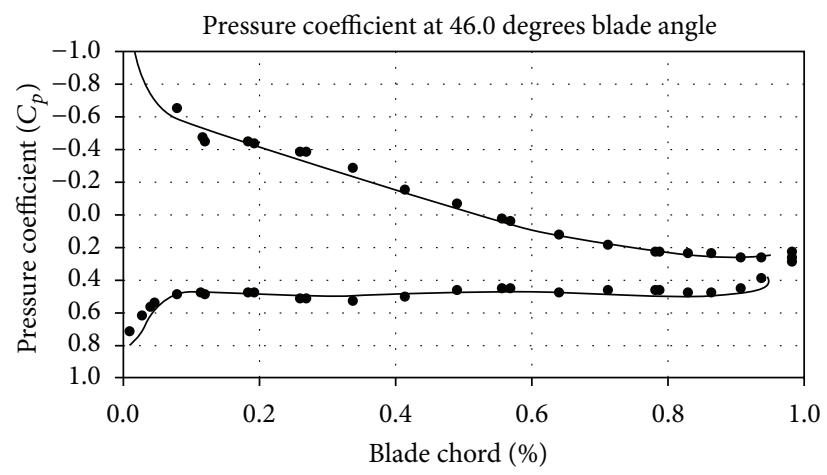

(c)

Figure 1: Two-dimensional Spalart-Allmaras computations of pressure coefficient for an isolated compressor blade (line) and three-dimensional measurements of pressure coefficient made in a compressor cascade at midspan (dots), from Rábai and Vad [20].

that one can model Reynolds stresses following a Bossiness approximation that utilises a single gradient diffusion hypothesis [18]:

$$
\overline{u_{i}^{\prime} u_{j}^{\prime}}=\frac{2}{3} k \delta_{i j}-v_{t}\left(\frac{\partial U_{i}}{\partial x_{j}}+\frac{\partial U_{j}}{\partial x_{i}}\right)
$$

which reduces the closure problem and requires an expression for eddy viscosity. One may model eddy viscosity using Prandtl's mixing length approximation [18]:

$$
v_{t}=\left|\frac{d u}{d y}\right|\left(\kappa y_{n}\right)^{2}
$$

which results in an accurate model for wall-bounded, attached flows with small pressure gradients. Although potentially useful in some applications, a modelling approach that is accurate with attached flows and small pressure gradients is not applicable in industrial fan applications where separated flow is inevitable. This lack of applicability is the primary drawback of a RANS approach. In practice, the models are derived from a series of simplifying assumptions that one can use to generate and calibrate coefficients using a series of benchmark cases. The benchmarking cases are not representative of the separated flow and large pressure gradients in industrial fans. This results in a very real risk that computational solutions do not adequately model the flowfield physics. This negative aspect that occurs with RANS solutions is unknown to many of those using commercial codes, and consequently they do not take the necessary care when setting up the solver.

The limitations that occur with using Prandtl's mixing length approximation are a reason why the majority of eddy viscosity models rely on one or more transport equations to derive a value for eddy viscosity. Spalart-Allmaras' oneequation model [19] has become the established eddy viscosity model, used in the majority of commercial codes. The industrial fan community typically utilises the Spalart-Allmaras model in two ways:

(i) computation of lift and drag coefficients of isolated aerofoils,

(ii) forming a baseline RANS closure for Detached Eddy Simulations (DES).

When studying the modelling of lift and drag coefficients, Rábai and Vad [20] reported that the Spalart-Allmaras model was capable of correctly predicting an isolated aerofoil's pressure distribution, lift and drag coefficients (Figure 1). Despite its success with isolated aerofoils, Šarić et al. [21] concluded that the model did not lend itself to application with the complex geometries that occur with internal flow calculations. In this context, we define an internal flow as a flow through turbomachinery blading, as opposed to the external flow that occurs with isolated aerofoil or aircraft wing flowfield predictions. 


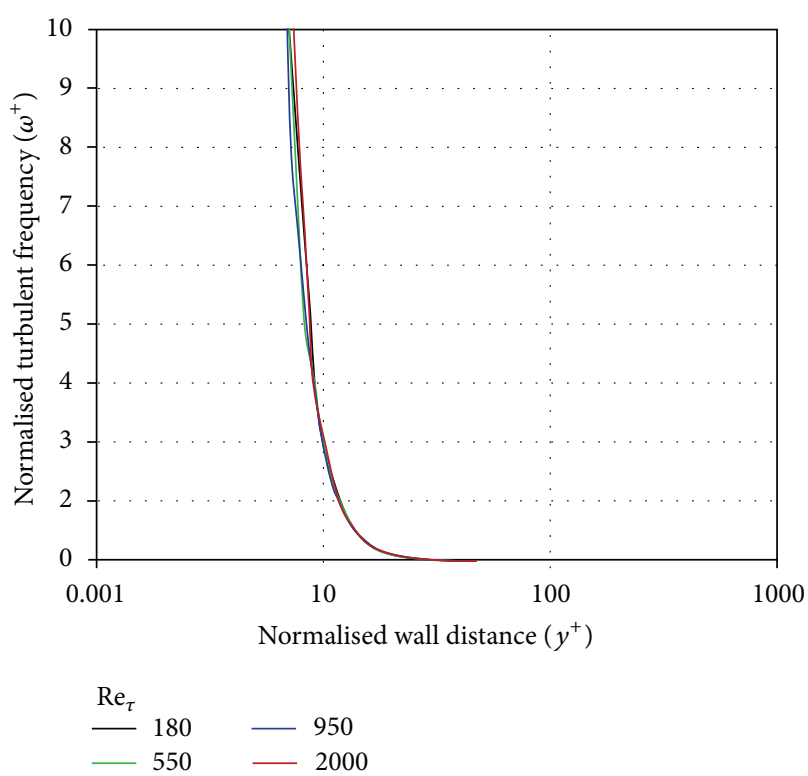

(a)

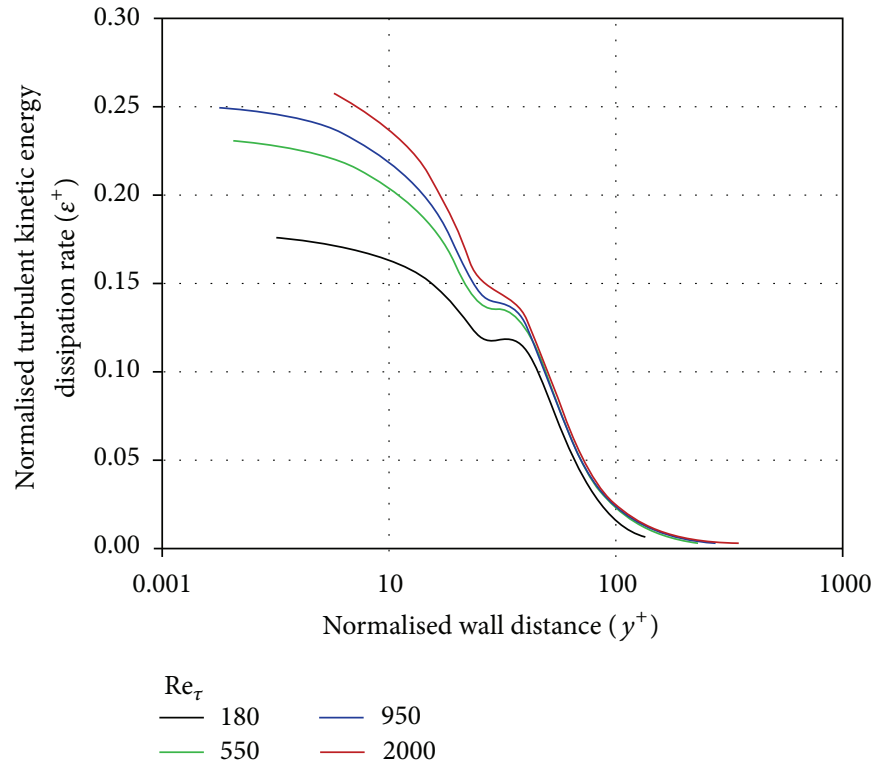

(b)

FIGURE 2: Channel flow computed using a direct numerical simulation (DNS). Normalised turbulent frequency $\left(\omega^{+}\right)$and normalised turbulent kinetic energy dissipation rate $\left(\varepsilon^{+}\right)$against normalised wall distance $\left(y^{+}\right)$calculated over a range of Reynolds number based on friction velocity $\left(\operatorname{Re}_{\tau}\right)$.

When dealing with internal flows, one can overcome the limitations of a one-equation model with a two-equation model. The currently favoured two-equation models are either the $k-\varepsilon$ model [22] or the $k-\omega$ model $[23,24]$. Both models require one to solve two transport equations to compute eddy viscosity as an algebraic expression of turbulent kinetic energy $(k)$ and dissipation rate of turbulent kinetic energy $(\varepsilon)$ or turbulence frequency $(\omega)$. In two-equation models the first equation is typically for turbulent kinetic energy $(k)$ and the second for either dissipation $(\varepsilon)$ or turbulence frequency $(\omega)$. Both the $k-\varepsilon$ model and the $k$ - $\omega$ model rely on an assumption that one can link eddy viscosity to a time and length scale that characterises turbulence that in turn links to the computed flow-field's characteristics. A feature of the $k-\varepsilon$ and $k-\omega$ models is that the additional transport equations for $k, \varepsilon$, and $\omega$ share the same form, and, therefore, for a generic $\phi$ quantity, it reads

$$
\frac{\mathrm{D} \phi}{\mathrm{D} t}=P_{\phi}-\varepsilon_{\phi}+\frac{\partial}{\partial x_{j}}\left[\left(\nu+\frac{\nu_{T}}{\sigma_{\phi}}\right) \frac{\partial \phi}{\partial x_{j}}\right] .
$$

On the left-hand side of (6) is the quantity's material derivative. On the right-hand side are one or more production terms, a dissipation term, a diffusion term dependant on molecular viscosity, and another given as the turbulent viscosity's function, corrected using the Prandtl number $\sigma_{\phi}$. The primary difference between the $k-\varepsilon$ and $k-\omega$ models is the different trend of $\varepsilon$ and $\omega$ at the wall and the definition of the wall boundary conditions for the same variables. When one studies normalised values of $\varepsilon$ and $\omega$ for an attached flow, it is evident that $\omega$ is less dependent on the Reynolds number than $\varepsilon$ in the wall's near vicinity (Figure 2).
There is a general consensus within the computational fluid dynamics community that the $k-\varepsilon$ model better reproduces the energy cascade of large-scale structures in the main flow core, whilst the $k-\omega$ model performs better near the wall [25]. A realisation that $k-\varepsilon$ models perform better in the main flow whilst the $k-\omega$ models perform better near the wall leads to the natural conclusion that, ideally, one would use the two models in combination. Hanjalić et al. [15] observed that it is possible to combine $k-\varepsilon$ and $k-\omega$ models as one can reformulate every two-equation model into every other by changing model coefficients. This realisation has enabled engineers to formulate the $k-\omega$ shear stress transport $(k-\omega$ SST) model [26] that solves the equation for $\omega$ near the wall and $\varepsilon$ elsewhere.

The use of two-equation models has become established within the industrial fan community. Pinelli et al. [27] reported a series of simulations with the objective of optimising a double inlet centrifugal fan's design by utilising a $k-\omega$ SST model. Pinelli et al. [27] were able to select the best geometry from three competing alternatives and correctly predict the pressure rise, volume flow rate characteristic curve (Figure 3). Corsini et al. [28] and Sheard et al. [29] reported a series of computations to predict the characteristic curve for an axial fan using a RANS simulation with a two-equation $k-\varepsilon$ model. Other researchers [30-36] report similar studies with two-equation models.

2.2. Wall Treatment. When studying and modelling the fluid mechanics of industrial fans, researchers have concluded that the key phenomena of interest are induced by large velocity gradients that occur with the presence of solid walls. When using a RANS approach to wall treatment, one can formulate 


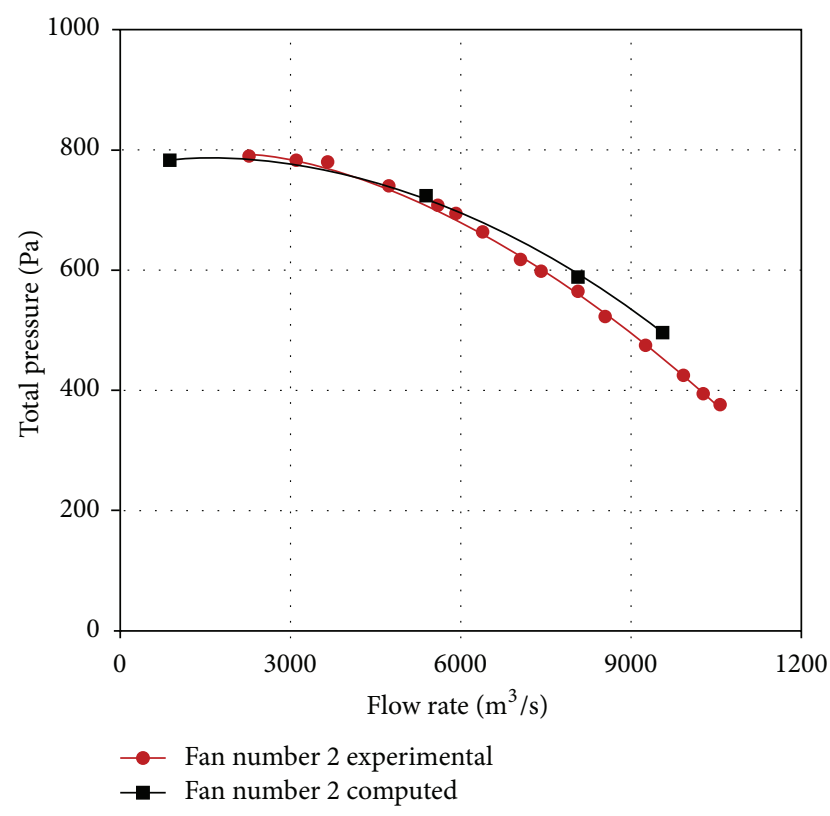

FIGURE 3: Characteristics computed using a RANS simulation with a $k$ - $\omega$ SST turbulence model and experimentally measured characteristics of a double-inlet impeller centrifugal fan, from Pinelli et al. [27].

every model into what we may characterise as either a highor low-Reynolds formulation. The difference between the two is integrating transport equations at the walls into the computational method.

When one studies the normalised velocity profile for channel flow at increasing Reynolds numbers, it is apparent that the profile is independent of Reynolds number. Researchers have studied the impact of Reynolds number on near-wall flow; $[13,14,17,18]$ have characterised the profile into three zones, a viscous sublayer for $y^{+}<5$, a logarithmic layer for $y^{+}>30$, and a buffer layer for the range of $5<y^{+}<$ 30 . It is the viscous sub-layer that is the most critical zone when modelling the flow-field (Figure 4).

The high-Reynolds formulation integrates transport equations into the computational method using wall functions that model fluid behaviour through the viscous sublayer to the wall. The first node of the computational grid is placed in the flow's fully turbulent region $\left(y^{+} \approx 40\right)$, reducing the grid density and therefore the required computational effort for a simulation. A drawback of this approach is that the wall function must be defined in a way that accounts for the wall's presence. Whilst this is practical, one typically derives wall functions from a series of assumptions:

(i) the boundary layer is attached,

(ii) the local turbulent energy is in equilibrium.

The above assumptions are not valid when the flow is separated, there is an adverse pressure gradient, or there is a wall curvature or impinging flow onto the wall. Those in the field inevitably associate industrial fans with separated flow. Their geometry induces adverse pressure gradients in the flow-field as a consequence of the fan blades' curvature. Impinging

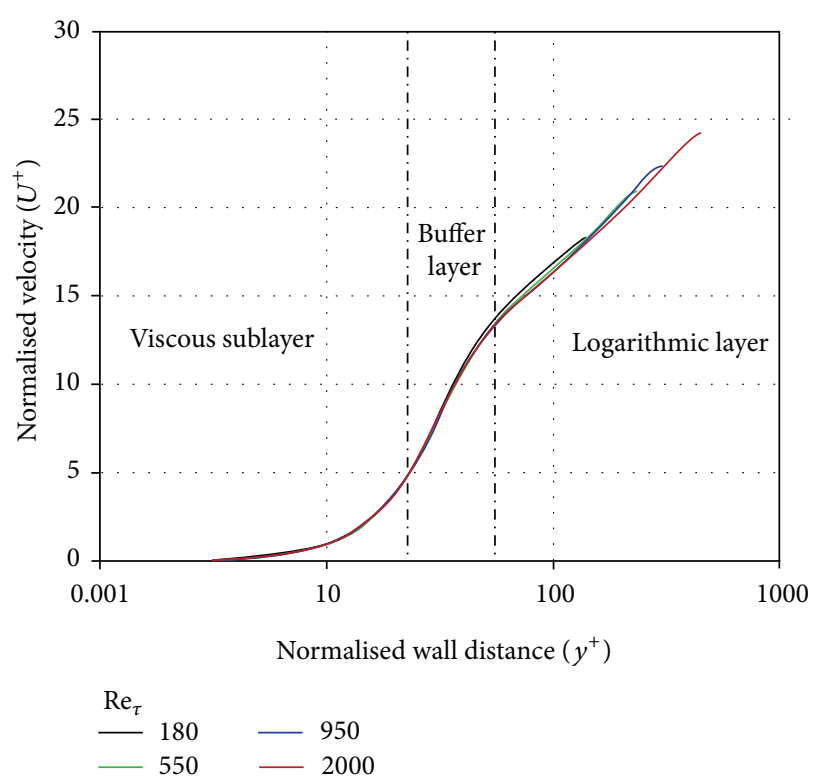

FIGURE 4: Channel flow computed using a Direct Numerical Simulation (DNS). Normalised velocity $\left(U^{+}\right)$against normalised wall distance $\left(y^{+}\right)$calculated over a range of Reynolds number based on friction velocity $\left(\mathrm{Re}_{\tau}\right)$ illustrating the range of normalised wall distance associated with the viscous sublayer, buffer layer, and logarithmic layer.

flow onto walls is inevitable because of blade tip-to-casing flow and blade wake impingement on static components. Consequently, the use of wall functions in a high-Reynolds formulation facilitates a reduction in the computational effort required for the simulation, but the wall functions themselves have their deficiencies.

A RANS model may address these deficiencies by integrating up to the wall. However, in order to integrate up to the wall, we must reformulate the RANS equations to account for viscous effects in the near-wall region. We may reformulate the equations by incorporating a logarithmic function of either Reynolds number based on friction velocity $\left(\mathrm{Re}_{\tau}\right)$ and normalised velocity $\left(U^{+}\right)$or normalised wall distance $\left(y^{+}\right)$and normalised velocity $\left(U^{+}\right)$. This makes it possible to account for the viscous effect in the viscous sub-layer (Figure 4). Davidson [14] provides a comprehensive description of the use of wall functions for high-Reynolds representations of (6) and their reformulation into a low-Reynolds form of (6).

2.3. Grid Refinement and Grid Independency. We may reformulate the RANS equations to at least partly account for viscous effects in the near-wall region by using a low-Reynolds formulation. When using a low-Reynolds formulation the mesh needs to be refined in the wall-normal direction. The objective is to ensure that the first grid node lies in the viscous sub-layer $\left(1<y^{+}<5\right)$. Both low- and high-Reynolds formulations place requirements on the computational grid. The low-Reynolds formulation requires the first grid node to lie in the viscous sub-layer. The high-Reynolds formulation requires placing the first node of the computational grid in 
the flow's fully turbulent region. However, it is not possible to know a priori if the first node lies in either the viscous sublayer or the flow's fully turbulent region. In practice industrial fan designers must rely on experience, using validated results from similar fan geometries operating over a range of similar mass flow rate. This experience informs two key choices:

(i) grid spacing near the solid boundaries (blades, hub and casing) in order to fulfil the requirements of either low- or high-Reynolds formulations,

(ii) estimating the number of cells required in order to achieve a grid independent solution.

When considering the required number of cells to achieve a grid independent solution, it is noteworthy that not all the computational domain parts are equally important. For example, we associate the blade tip-to-casing region with a tip-leakage vortex, and in order to model this flow feature, it requires a relatively high grid density. However, we must associate this cell clustering in regions of known flow-field features with a smooth transition from regions of low to high cell density. We must avoid cells with a large aspect ratio or those that are heavily distorted. Despite this caveat, we can minimise the required number of cells to achieve a grid independent solution through a grid refinement process, and therefore industrial fan designers routinely employ grid refinement.

One may also use grid refinement to cluster cells near to every wall. This maximises the probability that there will be a node in the viscous sub-layer and hence that a low-Reynolds formulation will accurately model the flow-field physics. A way to check that grid refinement has successfully resulted in a node in the viscous sub-layer is to adapt the RANS formulation, with the formulation now checking the $y^{+}$value during a simulation. If the $y^{+}$value is greater than the first node's distance from the wall, the RANS formulation dynamically switches from a low- to high-Reynolds formulation. Popovac and Hanjalić [42] characterised this dynamic switching from a low- to high-Reynolds formulation as a compound wall treatment. Popovac and Hanjalić [42] concluded that a compound wall treatment resulted in significantly more accurate computational simulation results.

A low-Reynolds formulation typically requires a grid with twice the number of required cells for a grid-independent high-Reynolds formulation. Despite the increase in computational effort that occurs with low-Reynolds formulations, industrial fan designers have judged the improvement in accuracy that occurs with low-Reynolds formulations to be more important than the increase in computational effort. Therefore, the majority of industrial fan designers use a RANS formulation with low-Reynolds formulation to predict overall fan performance over the stable part of the fan's characteristic.

2.4. Anisotropic Modelling of Eddy Viscosity. A limiting factor in a RANS formulation occurs with the definition of eddy viscosity, (5). Modelling eddy viscosity using Prandtl's mixing length approximation results in the Reynolds stress tensor aligning with the velocity's gradient. The Reynolds stress tensor is not aligned with the velocity's gradient in regions of separated flow or impingement, and therefore the modelling approach for eddy viscosity does not accurately model the flow-field physics. A method to improve the accuracy with which one models the flow-field physics without increasing the computational effort required is to use a nonlinear eddy viscosity model. Non-linear eddy viscosity models utilise a cubic definition of eddy viscosity $[37,43]$. A cubic definition of eddy viscosity results in the definition of Reynolds stresses in (4) assuming a more complex form:

$$
\begin{aligned}
\overline{u_{i}^{\prime} u_{j}^{\prime}}= & \frac{2}{3} k \delta_{i j}-v_{t} S_{i j} \\
& +c_{1} v_{t} \frac{k}{\varepsilon}\left(S_{i k} S_{k j}-S_{k l} S_{k l} \frac{1}{3} \delta_{i j}\right) \\
& +c_{2} v_{t} \frac{k}{\varepsilon}\left(\Omega_{i k} S_{k j}+\Omega_{j k} S_{k i}\right) \\
& +c_{3} v_{t} \frac{k}{\varepsilon}\left(\Omega_{i k} \Omega_{k j}-\Omega_{k l} \Omega_{k l} \frac{1}{3} \delta_{i j}\right) \\
& +c_{4} c_{\mu} v_{t}\left(\frac{k}{\varepsilon}\right)^{2}\left(S_{k i} \Omega_{l j}+S_{k j} \Omega_{l i}-S_{k m} \Omega_{l m} \frac{2}{3} \delta_{i j}\right) S_{k l} \\
& +c_{5} c_{\mu} v_{t}\left(\frac{k}{\varepsilon}\right)^{2}\left(S_{i k} S_{j l}-S_{k m} S_{l m} \frac{1}{3} \delta_{i j}\right) S_{k l} \\
& +c_{6} c_{\mu} v_{t}\left(\frac{k}{\varepsilon}\right)^{2} S_{i j} S_{k l} S_{k l}+c_{7} c_{\mu} v_{t}\left(\frac{k}{\varepsilon}\right)^{2} S_{i j} \Omega_{k l} \Omega_{k l} .
\end{aligned}
$$

The eddy viscosity's third order tensorial formulation in (7) partially accounts for surface curvature. The formulation is therefore better able to account for producing turbulent kinetic energy in impingement regions as it constitutes a more realistic anisotropic reproduction of Reynolds stresses with respect to linear eddy viscosity. Non-linear eddy viscosity models are a key feature of the formulation that enables a RANS simulation to simulate the flow-field in both turbomachinery [44] and fan applications [28]. Corsini et al. [36] illustrated the effectiveness of an advanced cubic $k-\varepsilon$ turbulence model. They were able to accurately predict the axial flow fan's characteristics within the uncertainty with which they could experimentally measure the fan's characteristics (Figure 5).

An alternative to a non-linear eddy viscosity model when accounting for the anisotropy of Reynolds stresses is the elliptic relaxation models $v^{2}-f[44]$ and $\zeta-f$ [45]. By solving two additional transport equations these elliptic relaxation models are able to account for the effect of wall blockage on Reynolds stresses and increase the RANS simulation's accuracy without a significant increase in computational effort [46].

Incorporating either a non-linear eddy viscosity model or an elliptic relaxation model constitutes an increase in complexity over that of a basic RANS formulation. The next level of complexity is the addition of second moment closure (SMC), also known as Reynolds stress models (RSM). The addition of SMC requires the RANS formulation to solve additional transport equations for the Reynolds stresses [4751] whilst correctly reproducing anisotropy. Durbin [52] 


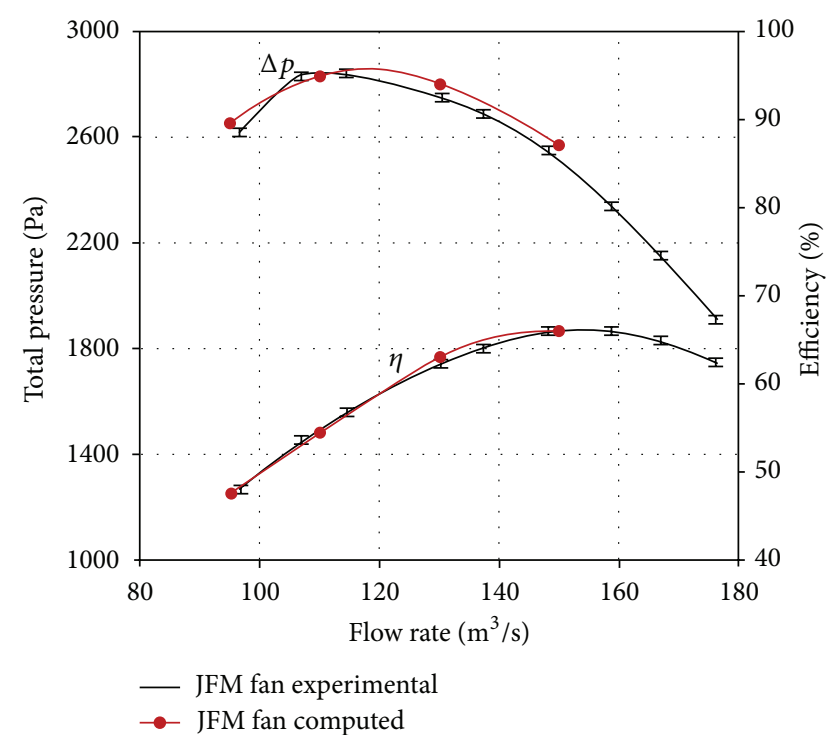

FIGURE 5: Numerical prediction and experimentally measured performance characteristics for a tunnel ventilation fan, from Corsini et al. [36]. Computations conducted using a low-Reynolds number cubic $k-\varepsilon$ model, from Lien et al. [37].

observed that the addition of second moment closure significantly increases a RANS simulation's accuracy when modelling the complex flow-fields that occur with turbomachinery applications $[49,53]$. A drawback of the addition of Second Moment Closure into a RANS formulation is the increase in computational effort as one must solve at least six additional transport equations. A further disadvantage is that the increase in numerical stiffness can lead to the solver's [51] nonconvergence, and, consequently, industrial fan designers do not favour RANS formulations with Second Moment Closure.

2.5. Rotation, Swirl, and Transition. As we discussed at the beginning of this paper, a drawback of a RANS approach is the assumptions on which the model relies. When studying physical transport phenomenon, Hanjalić et al. [15] observed that the equation for $k$ in RANS formulations is not sensitive to rotation, and that this is a primary deficiency of any RANS formulation. A way to improve a RANS formulation is to include terms into the formulation that account for the effect of rotation [54, 55]. Although potentially helpful, in practice these attempts to account for the effect of rotation have not improved the accuracy of results. In practice, the RANS formulations that give the best results are those that design engineers have adapted to the specific application [56, 57]. Therefore, a focus for a researcher working in the field is to identify specific RANS formulation deficiencies when applied into a specific application, with the objective of correctly reproducing the laminar to turbulent transition of boundary layers [57-60]. Ability to better model laminar to turbulent boundary layer transition is a key factor in modelling the blade-to-blade flow-field more accurately, and the subject remains an active research area.
The above provides an insight into the difficulty that occurs with using a RANS simulation to predict an industrial fan's blade-to-blade flow-field. The active research that occurs with tailoring RANS simulations to specific applications indicates that we cannot assume "standard" RANS models to give an accurate prediction of either an industrial fan's bladeto-blade flow-field or its overall performance. In essence, one must select or develop a RANS model to reproduce the flowfield physics relevant to each application. Pope [18] provides a good overview of the literature characterising RANS solution limitations, and the various ways in which design engineers have addressed those limitations.

\section{Unsteady RANS (URANS)}

A way to improve results with the Reynolds-averaged approach without adding application specific models is to solve the unsteady RANS equations. An unsteady RANS approach still does not account for the turbulence energy cascade from large-scale structures. However, an unsteady RANS is able to reproduce unsteady flow phenomena such as the blade tip-to-casing leakage vortex and the development of secondary flow features in the blade hub region. Piotrowski et al. [61] used an unsteady RANS approach and were able to compute the flow-field through a linear compressor cascade. They concluded that the blade tip-to-casing leakage vortex evolved over time, but with its position remaining fixed. In contrast, the hub secondary flow vortex did not remain in a fixed position. The hub secondary flow vortex development was characterised by shedding at a characteristic frequency. Yang et al. [62] studied the flow-field through a centrifugal fan using an unsteady RANS approach. He concluded that the predicted fan characteristic was more accurate using the unsteady RANS approach than has been possible previously using a steady RANS approach with the same $k-\omega$ SST turbulence model.

The primary drawback that engineers associate with an unsteady RANS approach when compared with a RANS approach is the increase in the required computational effort to compute a solution. The increase in computational effort is a consequence of the need to simulate the flow-field over a period of time to correctly model the time varying fluctuation of velocity, pressure, and flow-field features. Yang et al. [62] observed that an additional factor that impacts on the required computational effort for an unsteady RANS solution is the length of time step that one selects for the computation. The Courant-Friedrichs-Lewy (CFL) number limits the time step which is a necessary condition for convergence whilst solving partial differential equations numerically. It arises in the numerical analysis of explicit time-marching schemes. The time step must be less than a certain time; otherwise the simulation will produce incorrect results. The CFL number's limiting value is

$$
\mathrm{CFL}_{\max }<1
$$

If the CFL number remains below its maximum allowable value, the flow-field's time based resolution remains adequate 
for the mesh's spatial resolution that one uses for the computations. In essence, the finer the mesh, the smaller the maximum allowable time step in an unsteady RANS computation. Within the industrial fan community it is common practice to increase the time step such that $\mathrm{CFL}_{\max }<1.5$ without the accuracy of the resultant simulation degrading to the point at which conclusions that one draws from the results become error prone. However, a $\mathrm{CFL}_{\max }<1.5$ will only give valid results if the CFL number's average value over the entire computational domain remains below unity.

\section{Axial Fans}

The challenges that occur with computing both the blade-toblade flow-field and overall fan characteristic are application specific. We may split the applications into two basic groups, axial and centrifugal fans. First, we will consider axial fans.

4.1. Rotor-Only Computations, Pitchwise Periodicity, and Turbulent Structures. When modelling an axial industrial fan's blade-to-blade flow-field the least complex configuration that one can model is the rotor alone. If one neglects static components then one can carry out the computations in the rotating frame of reference, and, consequently, there is no requirement to account for the computational mesh's movement. Those researchers who have used a rotor-only approach augment the RANS momentum equation with terms that represent Coriolis and centrifugal forces [13, 14, 17, 18]. Denton and Dawes [63] and Denton [64] observed that one may use periodic boundary conditions at midpitch in order to compute the flow-field through a single blade or vane passage (e.g., see $[28,29])$.

A disadvantage that occurs with applying periodic boundary conditions is that they can fail to model accurately turbulent structures if one associates those structures with long wavelengths or length scales. The limitations that occur with applying periodic boundary conditions are not an issue with a RANS approach as the approach itself does not model the turbulent structures that periodic boundary conditions fail to model. Consequently, the use of periodic boundary conditions does not degrade the RANS simulation's accuracy.

An unsteady RANS simulation is able to model major unsteady blade-to-blade flow-field features [12, 61]. An example of a flow-field feature that an unsteady RANS simulation can model is the vortex shedding that occurs with blade wakes. Vanella [65] observed that when conducting an unsteady RANS simulation it can be advantageous to extend the computational domain to more than a single blade or vane passage. A reason for extending the computational domain when the studied fan has a high solidity is the possibility that turbulent structures that generate in one blade or vane passage may impinge upon the next. Consequently, an unsteady simulation that models only one blade or vane passage will not model the flow-field physics accurately.

It is only necessary to extend an unsteady RANS computational domain to the entire fan when attempting to gain insight into phenomena that affect the entire rotor. Vanella et al. [65] reported an example of phenomena that affects the entire rotor. They studied the onset of stall in an axial compressor using a Spalart-Allmaras unsteady RANS simulation, discretizing each rotor's blade-to-blade passage using a total of $74 \times 10^{6}$ cells. They considered this number of cells as the minimum required to adequately model the flow-field physics that occurs with rotor stall. The number of cells that Vanella et al. [65] used is approximately one order of magnitude larger than that which industrial fan designers routinely use and is consequently beyond that which is practical at the time of writing.

A further consideration when using a rotor-only simulation for either a RANS or unsteady RANS simulation is the computational domain's axial extent up- and downstream of the blade leading and trailing edge. For a fan with wellconditioned inflow characteristics, Corsini and Rispoli [28] concluded that one could achieve a mesh-independent solution with a computational domain that extended at least half a blade chord up- and downstream of the blade leading and trailing edge. When simulating the blade-to-blade flow-field for a fan with a distorted inflow, Thiart and von Backström [66] concluded that the computational domain must extend more than half a blade chord upstream of the blade leading edge, with the required distance dependent on the inflow distortion's extent.

A final consideration when using a rotor-only simulation for either a RANS or unsteady RANS simulation is the definition of inflow conditions. Engineers typically associate industrial fans with relatively low inlet flow velocities, and therefore it is reasonable to assume that the inflow is incompressible. It is therefore possible to define an inlet velocity profile and either a fixed turbulence level or a turbulence profile that accounts for the inlet velocity profile. A typical velocity profile would be the profile that engineers associate with fully developed pipe flow. A typical midstream turbulence level would be three percent, rising to ten percent in the near-wall region as velocity reduces [29].

When considering compressible flows an alternative approach is the fix static pressure at the outlet and total pressure and mass flow rate at the inlet. Hirsch [17] found this approach to result in an accurate simulation of the blade-toblade flow-field, if the computational domain was extended to more than one blade chord length forward of the blade leading edge. The assumption implicit in the assertion that outlet static pressure, inlet total pressure, and mass flow rate can be fixed is that the fan is operating in an ideal system. In practice, industrial fans are more usually operating in a complex system, and, consequently, heavily distorted inflow is the norm rather than the exception. When the inflow is heavily distorted an experimentally measured inflow velocity and turbulence profile are required. If experimentally measured inflow velocity and turbulence profiles are not available then one must add a portion of the inlet duct system to the simulation if the resulting solution is to be accurate.

4.2. Rotor-Stator Interaction and Contrarotating Fans. A practice that has become established in the industrial fan community is to prerotate the flow into an axial fan inlet [67]. Prerotating the air into the fan can facilitate a seven to ten percent increase in fan pressure developing capability with little or no reduction in fan efficiency. As an industrial fan motor 
is typically specified with between 10 and 15 percent reserve, adapting in-service fans to include the inflow's prerotation is a way to increase a fan's pressure developing capability. Increasing a fan's pressure developing capability may be required in the event that one incorrectly specified the fan which needs up to a ten percent increase in pressure developing capability in its application. One may model the effect of preswirl in a RANS or unsteady RANS simulation by distorting the inflow velocity profile to account for the pre-swirl effect [36].

When the required pressure developing capability exceeds that of a single fan by a factor of between two and two and a half, a common practice within the industrial fan community is to configure two fans, counterrotating in series [68]. The use of two counterrotating fans is common in tunnel ventilation systems, and engineers occasionally have adopted it in wind tunnel applications. An advantage of the counter rotating configuration is that the second fan removes the exit swirl from the first, resulting in axial flow downstream of the fan. A disadvantage is the relatively high blade passing velocity of the counter-rotating blades that result in two counter rotating fans producing approximately $15 \mathrm{~dB}$ more noise than one fan running alone. Modelling two counter rotating fans in either a RANS or an unsteady RANS simulation requires the model to account for the two blade rows' relative motion. We may model this in three ways, using a

(i) mixing plane,

(ii) frozen rotor, or

(iii) an unsteady coupling with moving mesh.

The mixing plane approach is the approach that researchers first developed when they attempted to model the interaction of counter-rotating blade rows or the interaction between a rotating and static blade row. The approach is based on the assumption that one could undertake separate steadystate simulations for each rotor or the rotor and the stator. One would then calculate circumferentially averaged velocity, pressure, and turbulence variables at the rotor's outflow and use these calculated values as the inflow conditions for the second rotor or stator [63].

A disadvantage of the mixing plane approach is that wakes that form the first rotor mix out at the mixing plane, rather than mix out gradually as they wash down-stream into the second rotor or the stator. Mixing out wakes instantaneously at the mixing place results in the inability of the mixing plane approach to simulate the wake's impact from the first rotor on the second rotor or stator. The effect of wakes from a rotor on a downstream stator is real, and one should model them. If one neglects them, the resultant simulation is still capable of predicting with reasonable accuracy the blade-to-blade flow-field and the rotor and stator's overall performance. However, this is not the case with a counterrotating fan. The effect of wakes from the first rotor on the second is significant enough for a mixing plane approach to reduce the second rotor blade-to-blade flow-field prediction's accuracy so far that it is no longer possible to accurately compute the overall fan performance.
A better approximation of rotor-rotor or rotor-stator interaction than the mixing plane approach is the frozen rotor approach. The primary advantage of the frozen rotor approach is that the rotor to rotor or rotor to stator coupling does not require a moving mesh. The relative position of the two rows is fixed in time, with relative motion managed using different frames of reference and adding Coriolis and centrifugal forces to the momentum equation in the rotating frame of [68]. In contrast to the mixing plane approach, the frozen rotor approach is able to account for a nonuniform circumferential distribution of velocity and pressure. Nonuniform circumferential distribution of velocity and pressure leads to a more realistic representation of wake mixing and, consequently, a more accurate simulation of the blade-toblade flow-field in the second rotor or stator.

Industrial fan designers have favoured the frozen rotor approach, as the resultant RANS or unsteady RANS simulations are able to predict the overall fan performance characteristic with good accuracy. This does not mean that the approach is without drawbacks. Adding Coriolis and centrifugal forces to the momentum equation in the rotating frame of reference constitutes an approximation of the flowfield physics that limits the resulting simulations' accuracy. To accurately simulate the flow-field physics it is necessary to undertake a fully unsteady coupling of the two blade rows. This requires a moving-mesh that can increase the required computational effort by up to an order of magnitude compared to a frozen rotor simulation. The increase in computational effort is a consequence of the required time to account for the interaction between the blade rows. The minimum number of interactions depends on clocking the two rows, with Yang et al. [62] proposing that computing one and a half rotor revolutions is sufficient to account for rotorstator interaction. Despite the advantages of a moving mesh approach, the additional computational effort associated with a moving mesh is the reason industrial fan designers have accepted the limitations of a frozen rotor approach.

\section{Centrifugal Fans}

The modelling considerations that apply to an axial fan are unchanged for a centrifugal fan, with one exception. When modelling centrifugal fans, one cannot reduce the computational effort by modelling a single blade passage with imposed periodic boundary conditions. Centrifugal fan simulations must be full rotor simulations, and, consequently, they inherently require more computational effort than an axial fan single blade passage flow-field simulation.

Issues with inflow and outflow boundary conditions are similar for both axial and centrifugal fans. However, centrifugal fans require additional consideration of the computational domain's extent up- and downstream of the rotor. At the fan inlet it is customary to add a hemisphere with a diameter two to three times the fan inlet's diameter or location at which total pressure is specified $[38,69]$. The fan outlet is complicated because centrifugal fans are typically coupled with an exhaust volute that discharges through a diffuser. Consequently, to correctly predict a fan's characteristics it is customary to extend the computational domain three duct 
diameters down-stream of the diffuser tongue. Fan mass flow rate is then specified at the down-stream limit of the computational domain $[38,69]$.

With a centrifugal fan simulation it is not possible to use the mixing plane approach when modelling the fan rotor and stator. The mixing plane approach relies on circumferentially averaging velocity and pressure. A high circumferential pressure imbalance characterises centrifugal fans, and, therefore, any simulation must be capable of modelling this unbalance [38]. Within the industrial fan community the established approach for modelling a centrifugal fan impeller is to use the frozen rotor approach. Researchers have used the frozen rotor approach using both RANS and unsteady RANS simulations to predict centrifugal fan characteristics. Son et al. [70] utilised a RANS simulation with frozen rotor and $k-\varepsilon$ turbulence model in high-Reynolds formulation to study the effect of varying inlet bell mouth geometry on a centrifugal fan's flow rate characteristics.

In practice, an unsteady RANS simulation is best able to simulate the flow features that occur with fan stall. Yang et al. [62] was able to use an unsteady RANS simulation to predict a centrifugal fan's characteristics up to its stall point. Lee et al. [38] were able to demonstrate that a high-Reynolds number formulation, standard $k-\varepsilon$ turbulence model, and frozen rotor approach proved to be sufficient to correctly predict a large centrifugal fan's characteristics for process industry applications both at peak efficiency and at peak pressure operating points (Figure 6).

\section{Simulation of Ventilation Systems with Synthesized Methods}

The computational methods that we have discussed so far have been concerned with predicting the blade-to-blade flowfield through the blade passage of either axial or centrifugal fans. In a development environment the objective is to facilitate a prediction of the fan's characteristic for either an existing geometry or a proposed new geometry. Although the computational methods can account for inflow and out-flow boundary conditions, the simulations are essentially standalone fan simulations. One does not account for the system within which the fan is to be embedded.

The majority of industrial fans are embedded into a system. These systems may be complex, with multiple fans and dampers interacting and, consequently, inducing significant variations in fan inflow and out-flow conditions. As industrial fans must operate in a system, there is a need to be able to predict the system's performance and the impact of that system on the fans embedded within it. However, the computational approaches that work well when modelling an axial or centrifugal fan's blade-to-blade flow-field are not suitable for modelling a system. A fan's characteristic time scale is invariably related to the fan's rotation speed, with high speed centrifugal blowers operating at up to 10,000 rpm. In contrast, the complex systems within which industrial fans are embedded have characteristic time scales related to the speed of sound, and, consequently, in large systems the system's characteristic time scale is typically between one and ten seconds.

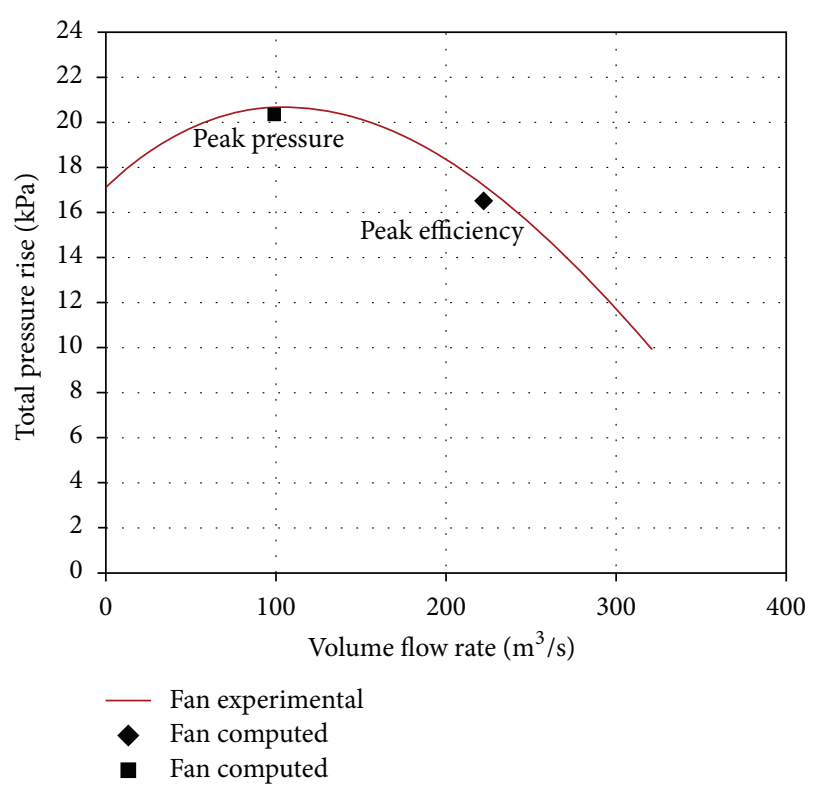

FIGURE 6: Numerically predicted and experimentally measured characteristic of a centrifugal fan originally developed for application in the cement industry. Computation conducted using a highReynolds number standard $k-\varepsilon$ turbulence model and a frozen rotor approach, from Lee et al. [38].

To model the flow-field accurately through a system the computation must extend over a time period longer than the system's characteristic time scale. In practice, this would require one to calculate the fan's blade-to-blade flow-field over hundreds or thousands of revolutions. The required computational effort to calculate a blade-to-blade flow-field over so many revolutions is orders of magnitudes greater than that available to industrial fan designers. Consequently, the computational methods that they apply to an axial or centrifugal blade-to-blade flow-field's predictions are not suitable for predicting system performance. Therefore, one must predict system performance by synthesising the fan's effect on the system within which it is embedded.

6.1. Fans as Pressure Discontinuities. When considering how to model the fan's impact on the system within which it is embedded the least complex approach is to treat the fan as a discontinuity of static pressure. This requires knowing the fan's pressure and volume characteristic, such that one may relate correctly the flow rate through the fan to the pressure discontinuity across it. The system model then treats the fan as a surface or single layer of cells across which it applies the discontinuity. In this way, the effect of a fan on a system may be synthesised using a "volume condition." Angeli [71] used the volume condition approach to synthesise the presence of jet fans in the Mont Blanc tunnel. This simulation's aim was to model the entire Mont Blanc tunnel system to enable the impact of different tunnel operating scenarios on the average air velocity induced in the tunnel by the jet fans. In practice, jet fans are required to induce an average air velocity of at least 
three metres per second if they are to clear smoke and keep escape routes clear in the event of a fire.

6.2. Actuator Disc and Actuator Line Methods. The least complex approach to modelling a fan installed in a system is the volume condition approach. More complex approaches are the actuator disc and actuator line approaches. Betz [72] developed these approaches for modelling wind turbines. Engineers have now applied these approaches to fans when installed in a system [73]. They involve the introduction of an additional body force in the momentum equation that accounts for the exchange of momentum between blades and fluid. They require that the computational method has incorporated into its code lift and drag coefficients for the fan blades at a series for spanwise blade sections. Engineers use the computational method to compute the incoming fluids angle of attack at each blade spanwise location. They then use the angle of attack in combination with the lift and drag coefficients to compute the additional body force on the fluid, following which the code is able to redistribute the exchange of momentum within the fluid.

More sophisticated codes are able to compute the exchange of momentum between the blades and fluid at runtime using the boundary element method (BEM) to dynamically recompute the blade's lift and drag as a function of the current velocity profile. With the actuator disc approach the exchange of momentum is treated as constant at different radial positions and not dependant on the azimuthal coordinates [72]. Meyer and Kröger [73] utilised the actuator disc approach to successfully predict a fan's characteristics. They also reported that the approach resulted in a slight underprediction of fan power consumption that they attributed to the approach's inability to account for tip-clearance phenomena. Moreover, the streamwise velocity profile downstream of the fan was slightly underpredicted due to the modelling assumptions. Despite these reservations, van der Spuy et al. [39] were able to use the actuator disc approach to accurately simulate the performance of an array of 288 axial flow fans (Figure 7).

The actuator line approach is similar to the actuator disc approach. However, unlike the actuator disc approach, the actuator line approach considers every fan blade and the fan blades' rotation within the computational domain. Sørensen and Shen [74] originally developed the actuator line approach for modelling wind turbines. The approach involves mapping each blade with a series of actuator points along actuator lines. One computes body forces at each point using the actuator disc approach methodology. One then applies the body force at the actuator point via a three-dimensional Gaussian function to avoid singularities. As the actuating lines rotate inside the computational domain, it is possible to both account for unsteady wake release and simulate the rotating blades' interaction with a stator.

\section{Modelling of Erosion}

Erosion of both axial and centrifugal fan blades is a major issue for industrial fan designers. Engineers classically associate axial fans in induced draft power and centrifugal fans in

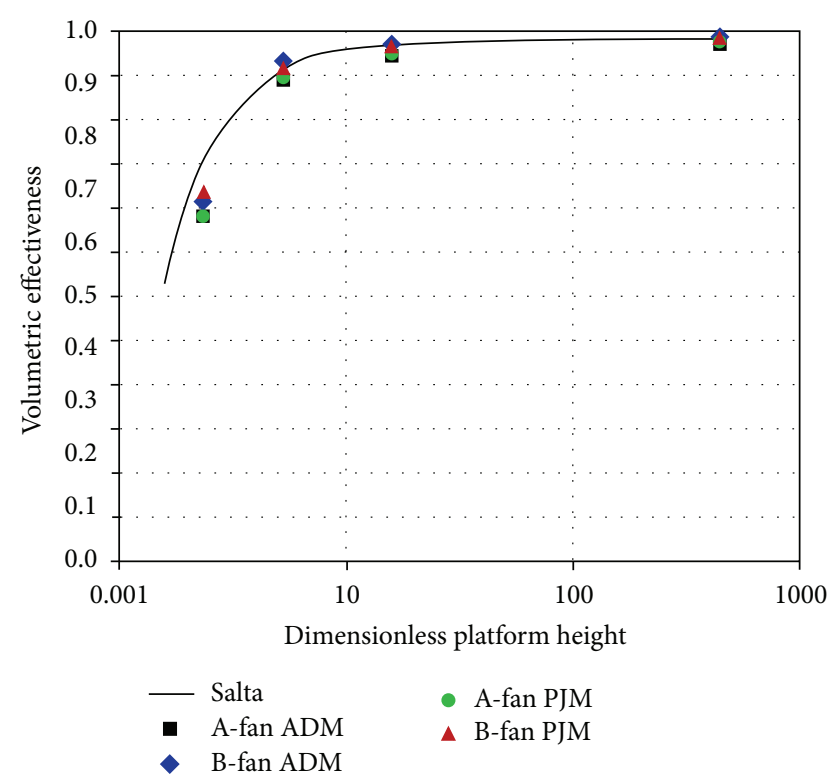

FIGURE 7: Numerical predictions of the volumetric effectiveness of an array of fans (symbols) and experimental measured performance of the array (line). Computations based on the synthesised actuator disk model approach, from van der Spuy et al. [39].

cement application with gas passing through the fan containing erosive particles. These particles both change the aerodynamic profile and reduce the fan blades' chord length. The combined effect is a reduced fan pressure developing capability and efficiency. Predicting erosion patterns is critical during blade design optimisation to minimise susceptibility to erosion and, subsequently, to predict the rate of material loss and, consequently, the fan maintenance schedule. Therefore, developing computational methods that can predict erosion is critical when developing new industrial fans intended for application in erosive environments.

We may achieve numerical prediction of erosion in one of two ways: Lagrangian computation of particle dispersion via single particle tracking [75] or via cloud particle tracking [76]. Single particle tracking involves computing single particle trajectories at runtime using the calculated flowfield parameters at the particle's current location. In contrast, cloud particle tracking involves tracking a statistical representation of an entire particle cloud, only computing the cloud's centre trajectory. Single particle tracking is the more accurate approach. However, Venturini [75] observed that the computational effort required for single particle tracking is between one and two orders of magnitude larger than that required for cloud particle tracking. For this reason, industrial fan designers favour cloud particle tracking.

Once one has computed the particle trajectory, either via single particle tracking or via cloud particle tracking, erosion may be predicted by considering the velocity and angle at which particles impact solid walls. Erosion is a function of velocity, angle of impact, and impact energy.

Tabakoff et al. [77] developed an erosion model that Corsini et al. [40] applied in a RANS code. They were able to successfully predict the axial fan blade's erosion rate in an 


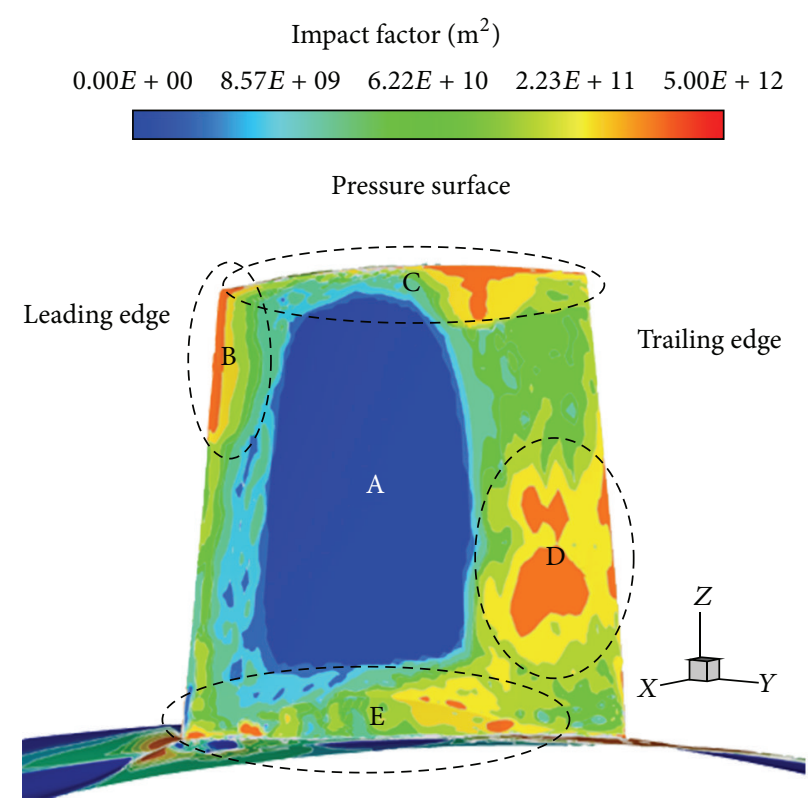

(a)

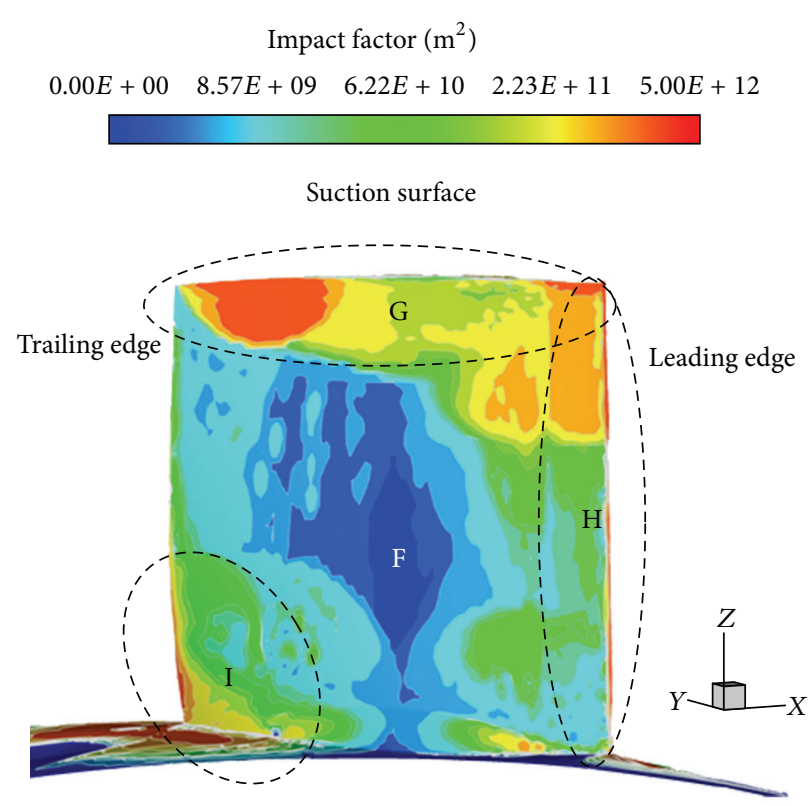

(b)

FIGURE 8: Global impact frequency after 10,000 hours of operation: pressure side (a) and suction side (b), from Corsini et al. [40].

induced draft power plan fan (Figure 8). Corsini et al. [40] were able to identify the blade pressure surface area (A) and suction surface area (F) that particle impact did not affect. Additionally, they were able to identify the pressure surface areas which particle impact most affected. These included the pressure surface outer 20 percent of blade span at the leading edge (B), an area affected by the tip leakage vortex (C), an area close to the blade trailing edge (D), and areas close to the blade hub (E). In contrast, the suction surface was primarily affected in the blade tip region $(\mathrm{G})$, along the entire blade span at the leading edge $(\mathrm{H})$, and at the trailing edge in the near-hub area (I). Ghenaiet [78] also used Tabakoff et al's [77] erosion model to study the effect of sand ingestion into a ventilation system. Consequently, we may conclude that although predicting erosion is challenging, it is possible, and the particle cloud tracking approach provides industrial fan designers with a practical method for doing so.

\section{Flow Induced Vibrations and Computational Aeroacoustics}

The success of those researchers who have used computational methods to predict erosion illustrates that computational methods have progressed beyond an isolated prediction of the blade-to-blade flow-field through a fan rotor. This progress manifests itself in two primary areas of research, the computation of aeroelastic induced vibration and computation of overall noise level and noise spectrum. Using the three-dimensional computational fluid dynamic code of $\mathrm{He}$ and Denton [79] as a basis for predicting the blade-toblade flow-field, researchers have calculated the flutter of turbomachinery blades. A full discussion of the application of computational methods to both the prediction of aeroelastic induced vibration and overall noise level and noise spectrum is beyond the scope of this paper. A comprehensive review of aeroelastic computational methods as applied to turbomachinery is provided by Marshall and Imregun [80]. A comprehensive review of computational aeroacoustics (CAA) is provided by Envia et al. [81].

\section{Future Trends}

When modelling the physics of all turbomachinery flow, RANS approach limitations hinder the accuracy with which one can model the blade-to-blade flow-field. Consequently, a RANS approach limits the accuracy with which one may calculate industrial fan performance characteristics. The RANS approach is not able to model the turbulence's spectral content and is not sensitive to the flow-field's multiscale features. Scholars who have studied the flow-field physics within all turbomachinery classes are increasingly turning to large Eddy simulations (LES) to overcome RANS approach limitations.

The principle issue with all large Eddy simulations is the formidable computations effort. The computational effort is simply beyond the reach of even the largest industrial fan manufacturers at the time of writing. The required computational hardware to successfully run a large Eddy simulation is between two and three orders of magnitude greater than that currently available to industrial fan designers. Despite this reservation, high performance computing (HPC) facilities are developing rapidly, exploiting massively parallel clusters of individual processors. Despite the promise of the high performance computing facilities, at the time of writing the costs to use them is beyond the reach of industrial fan manufacturers.

Further, the use of high performance computing facilities requires that an expert rewrites and optimises the 
computational methods to run across thousands of individual processors. To further compound matters, the commercial CFD code industry sells commercial CFD codes on a "per processor" basis that effectively makes the cost of running the codes across more than a handful of processors prohibitive. We may therefore conclude that it is likely to be another five years before industrial fan manufacturers can realistically consider a large Eddy simulation approach to predict a fan's performance characteristic.

Despite the challenges that large Eddy simulation poses, turbomachinery designers are moving towards large Eddy simulation based computational methods. This movement has been difficult, in part, as a consequence of Large Eddy Simulation based computational methods requiring a higher level of user capability than RANS based computational methods. However, Large Eddy Simulation based methodologies offer the possibility to compute unsteady phenomena, such as those that occur with stall inception, unsteady blade and bearing loads, vibration, and noise. The industrial fan community widely anticipates predicting the unsteady flowfield parameters responsible for generating overall fan noise and spectrum. A computational method that could give a reliable prediction of not only a fan's aerodynamic characteristics, but also acoustic characteristics would be useful during new fan design, development, and optimisation.

Although full Large Eddy Simulation methodologies are still some years away from routine application within the industrial fan community, there is progress with "hybrid" approaches. These hybrid approaches are based on sensitising an unsteady RANS approach to instabilities. Notable examples are detached Eddy simulations (DES) and scalaradaptive simulations (SAS). Other hybrid methods utilise a combined Large Eddy Simulation and RANS approach, with the actuator disc and actuator line approaches also offering the possibility of combining with a Large Eddy Simulation approach.

The detached Eddy simulation approach is a sensitised Large Eddy Simulation method based on the unsteady RANS Spalart-Allmaras model. The Detached Eddy Simulation approach is the most widely used of the available hybrid methodologies as it is able to account for the turbulence spectral content in turbomachinery simulations. At the time of writing, industrial fan designers have not reported in the literature the use of the Detached Eddy Simulation approach. Therefore, we may conclude that the computational effort that engineers associate with a Detached Eddy Simulation approach remains beyond that available within the industrial fan community at the present time.

The scalar-adaptive simulation approach is similar to the Detached Eddy Simulation approach as it utilises a methodology based on an unsteady RANS methodology [82-84]. The Scalar-Adaptive Simulation approach is based on a $k-\omega$ SST turbulence model sensitised to more than one characteristic length scale by introducing second order derivatives of velocity. Yang et al. [62] utilised the Scalar- Adaptive Simulation approach to compute the flow-field through a centrifugal fan to facilitate the study of unsteady loads on the fan impeller and to predict the pressure fluctuation magnitude. Wolfram and Carolus [69] also used the Scalar-Adaptive Simulation approach to compute the flow-field through a centrifugal fan. They were able to identify vortical structures that developed in the fan inlet and went on to study the mechanism by which these vortical structures entered the fan impeller and their role in generating the fan's acoustic signature.

Finally, hybrid Large Eddy Simulation/RANS methodologies solve the unsteady RANS equations in the near-wall region and the Large Eddy Simulation equations in the flow's main core. This hybrid approach reduces the required computational effort for a full Large Eddy Simulation whilst still accounting for large-scale structures in the majority of the flow. This models a part of the turbulence spectrum. Piotrowski et al. [61] observed that this hybrid approach is facilitated as the discretised system of Large Eddy Simulation and RANS equations share the same form, and, consequently, bridging the two within a single computational code is relatively easy to accomplish.

A challenge of using a hybrid Large Eddy Simulation/ RANS methodology is the interface between the two. Borello et al. [85] studied the practicality of interfacing Large Eddy Simulations and RANS computations in a single hybrid simulation. They studied the relative merits of both a moving and a fixed surface to create a buffer zone between the Large Eddy Simulation and the RANS computations, with the buffer zone thickness comprising typically three to five cells. Borello et al's hybrid approach [85] is noteworthy in that it is based on an elliptic relaxation RANS model. They demonstrated that this model could correctly reproduce the tip leakage vortex in a linear cascade without an increase in grid resolution when compared to an unsteady RANS computation based on the same RANS closure. Consequently, the required computational effort for the hybrid Large Eddy Simulation/RANS computation does not increase in comparison to an unsteady RANS because of mesh density. However, the hybrid Large Eddy Simulation/RANS computation does require more throughflow time steps than the unsteady RANS computation to achieve convergence, and one must reduce the time step amplitude to maintain the CourantFriedrichs-Lewy (CFL) number below unity. As a result, a hybrid Large Eddy Simulation/RANS simulation required approximately twice the computational effort of a comparable unsteady RANS simulation.

In theory, one can couple the actuator disk and actuator line approaches with a RANS or Large Eddy Simulation computation. This would allow the resulting hybrid simulation to reproduce large-scale structures' unsteady interaction. Sørensen and Shen [74] noted that at the present time there are no examples of hybrid methodology applications based on either the actuator disk or actuator line approaches applied to fans. However, engineers have successfully applied both approaches to wind turbines and therefore have the potential to apply them into industrial fan applications.

Finally, Davidson [86] noted that a theoretically possible hybrid approach would be to use low-resolution grids coupled with advanced subgrid scale models. Borello et al. [41] were able to demonstrate the potential of this approach by incorporating a subgrid scale model sensitive to each grid cell's size. Using this approach, Borello et al. [41] were able to recover part of the turbulence spectrum that is lost with 


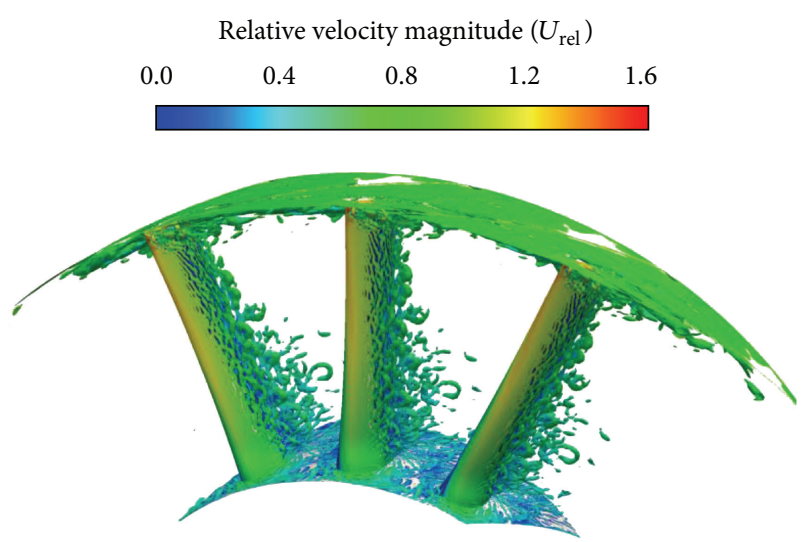

FIGURE 9: Numerically predicted vortical structures through the blade passage of a tunnel ventilation fan, computed using a Large Eddy Simulation (LES). These structures are visualised with isosurfaces of vortical structure deduction parameter $(Q)$ and coloured with relative velocity magnitude $\left(U_{\text {rel }}\right)$, from Borello et al. [41].

a RANS approach and were able to analyse the turbulent structures within a tunnel ventilation fan's blade-to-blade passage (Figure 9).

As Borello et al.s methodology [41] is inherently unsteady, they were able to utilise it to study the effects of train induced pressure pulses on tunnel ventilation fans. Borello et al. [41] concluded that the quasi-instantaneous changes of the operating conditions did not influence predicted large-scale turbulent structures. In contrast, the operating condition's quasi-instantaneous changes did impact small scale turbulent structures (Figure 10). However, Borello et al's [41] most significant conclusion was that the operating condition's quasiinstantaneous changes resulted in doubling the unsteady blade loads, despite the fan not driving into stall (Figure 11). They concluded that doubling the unsteady blade load was likely the cause of recent tunnel ventilation fans' in-service mechanical failures in tunnel systems with large pressure pulses.

Authors of commercial CFD codes do not champion hybrid methodology development. They are slow to respond to market demands for more advanced hybrid methodologies, preferring instead to exploit a monopolistic position in the market with well-proven RANS based methodologies that converge reliably. As a direct result the last decade has seen the widespread adoption of open-source codes. Foremost, amongst the open-source CFD codes that industrial fan designers have adopted are OpenFOAM [87] and CODE_ SATURNE [88]. These open-source codes are free and customisable. The industrial fan community is fragmented, with the majority of industrial fan designers working in small to medium size organisations that do not have the financial resources to pay the multiprocessor licence fees associated with commercial codes. Additionally, industrial fan designers typically develop products for a wide range of applications, and, consequently, the ability to customise a code is attractive.

The current generation of open-source codes meets the scalability standards of large High Performance Computing facilities, and, consequently, they can take advantage of rapidly developing massively parallel computation capabilities. An additional advantage of open-source computational fluid dynamic codes is that one can couple them with other codes. For example, the open-source optimising code DAKOTA [89] is able to operate in conjunction with a computational fluid dynamics code, passing back a revised set of parameters to optimise blade geometry through a Pareto optimisation study. When attempting to optimise an aerofoil, Spisso [90] combined OpenFOAM and Point Wise, a commercial mesh generator with built-in scripting capabilities for automated mesh generation and DAKOTA. The industrial fan industry has a long history of producing either design-toorder or heavily adapted-to-order fan designs. Therefore, the emergence of viable optimisation tools represents an exciting development that is likely to become increasingly relevant to industrial fan designers over the next five years.

\section{Conclusions}

Computational methods have become established within the industrial fan community. Despite the inevitability of separated flow through industrial fans, engineers have developed computational methods that simulate both the blade-to-blade flow-field and overall fan performance and to an accuracy that is of practical use to industrial fan designers.

For the first time, the legislative environment within Europe and the planned legislative environment in the USA are setting minimum fan and motor efficiency levels. This change in legislative environment is driving industrial fan designers to adopt computational methods as they are now legally required to improve fan performance to at least the legal minimum. This legislative imperative is resulting in industrial fan designers adopting open-source code in an ongoing effort to spread the use of computational codes from analysis based research applications into development and order related engineering application. Within the next five years it is likely that the industrial fan community will have abandoned commercial codes in favour of open-source alternatives.

10.1. CFD as a Design Tool. Today the industrial fan community routinely uses computational fluid dynamic codes as design tools. Commercial codes incorporating a basic RANS methodology with either a $k-\varepsilon$ or $k-\omega$ wall function approach are able to predict both axial fan and centrifugal fan performance characteristics. Over the stable part of the fan's characteristic these predictions are accurate enough to be of practical use to industrial fan designers. Further, they achieve the predictions with a computational effort that is realistic for the majority of industrial fan designers.

As either an axial or centrifugal fan approaches peak pressure, and therefore its stability limit, classical RANS based methodologies with wall functions reach their capability limit. The hypotheses that engineers used to derive all RANS methodologies are no longer valid. As a fan approaches stall, flow-field structures become more complex, and, consequently, it is no longer possible to accurately predict the fan's performance characteristics. 

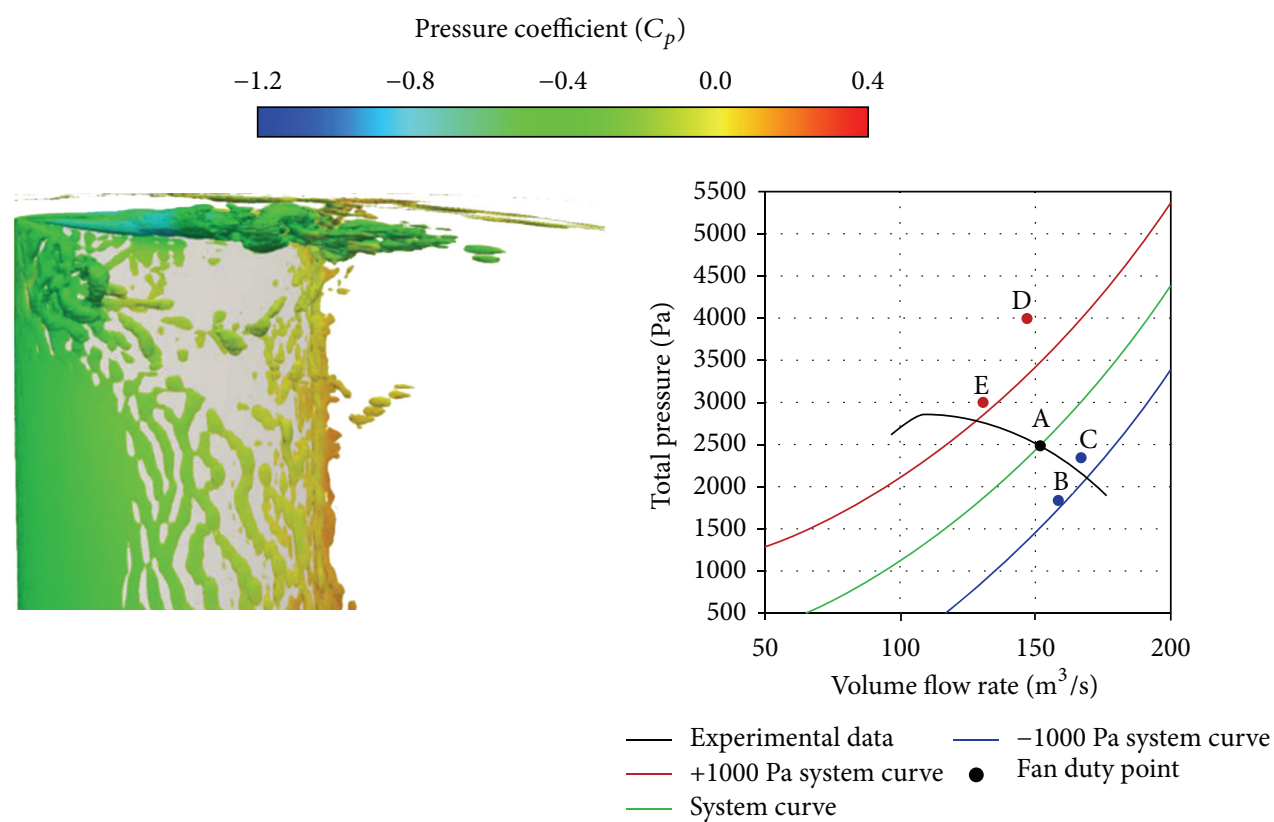

(a)

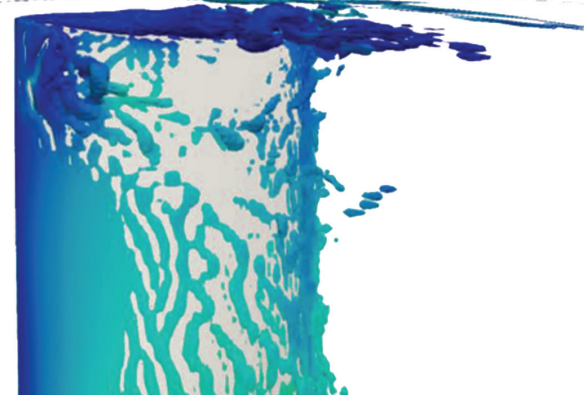

(b)

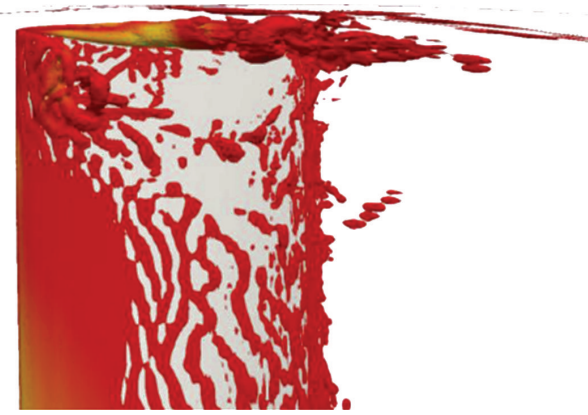

(d)

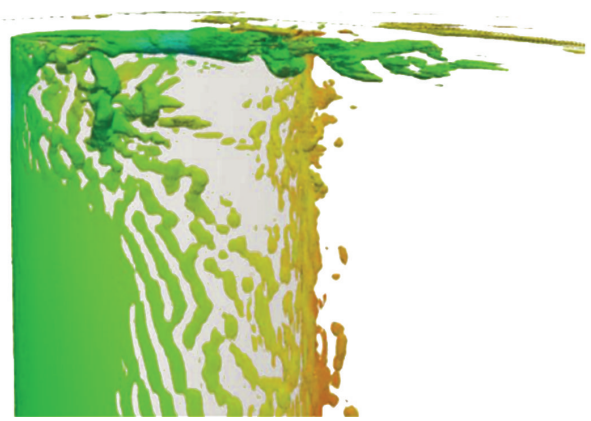

(c)

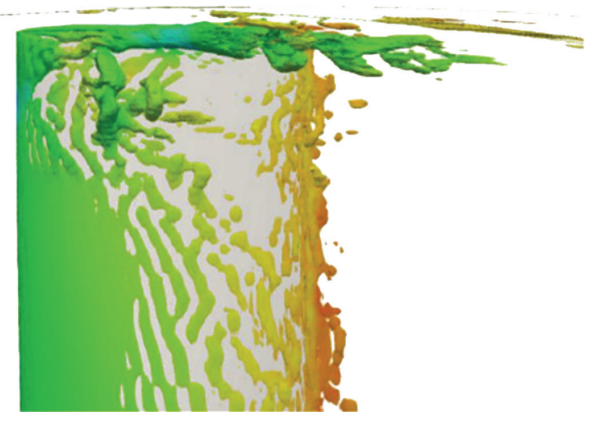

(e)

Figure 10: The numerically predicted vortical structures through a tunnel ventilation fan's blade passage, computed using a Large Eddy Simulation (LES). These structures are visualised with isosurfaces of vortical structure deduction parameter $(Q)$ and coloured with pressure coefficient $\left(C_{p}\right)$, from Borello et al. [41].

Despite the inability of RANS based methodologies to predict a fan's characteristic when stalled, the numerical prediction is still able to identify that the fan is stalling. Industrial fan designers are primarily focused on ensuring that their designs do not operate in stall. Consequently, it is enough for a computational method to predict that the fan will stall, and that is why RANS based methodologies remain the favoured methodologies within the industrial fan community.
If the computational method is required to predict a fan's characteristic performance when stalled, a methodology that better models the flow-field physics is required. Moving from a high- to low-Reynolds formulation in the RANS methodology improves the flow-field physics' modelling. However, a low-Reynolds formulation requires a substantial increase in grid resolution close to walls. Consequently, a low-Reynolds formulation typically requires twice the computational 


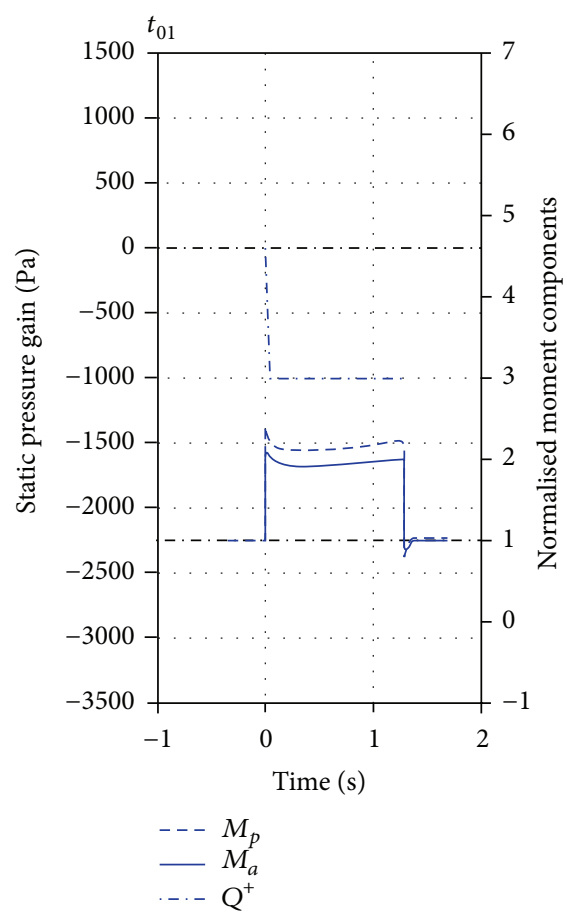

(a)

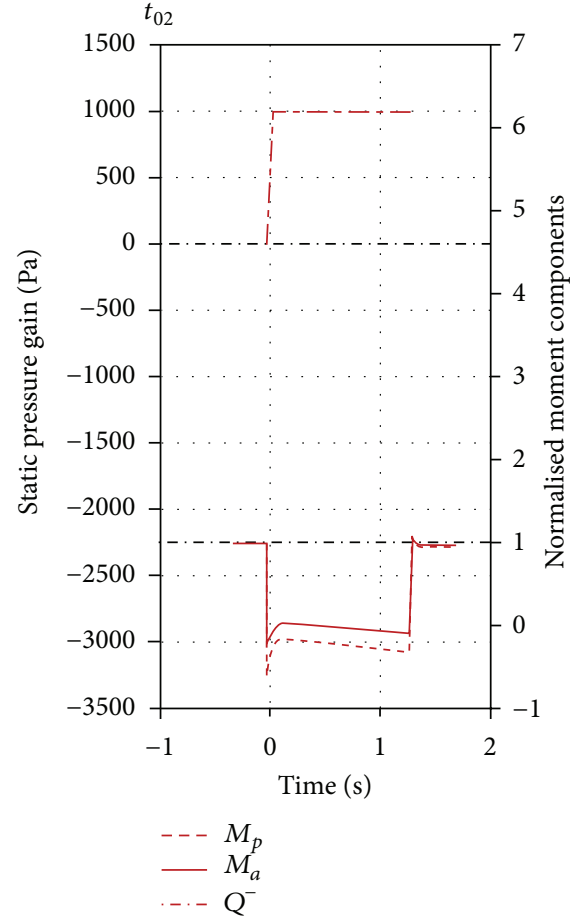

(b)

FIGURE 11: Time histories of the axial and peripheral moment components $M_{a}$ and $M_{p}$ on the blade surface under the effects of increasing $\left(Q^{+}\right)$ and decreasing $\left(Q^{-}\right)$volume flow rates. Values are normalised with respect to the corresponding value associated with normal fan operation at its nominal duty point, from Borello et al. [41].

effort required for a high-Reynolds formulation. Despite the increase in computational effort, today industrial fan designers favour RANS methodologies with a low-Reynolds formulation.

10.2. CFD as an Analysis Tool. Computational fluid dynamics codes offer industrial fan designers the possibility of simulating in detail the blade-to-blade flow-field. Codes incorporating a basic RANS methodology predict a fan's overall performance characteristics well, but the limitations inherent in their formulation make them less suitable for studying the blade-to-blade flow-field. Real insight requires a code based on an unsteady RANS methodology incorporating advanced low-Reynolds models, a Detached Eddy Simulation (DES) or a Scalar-Adaptive Simulation (SAS). These unsteady RANS based methodologies have the potential to simulate the blade-to-blade flow-field as a fan approaches stall. They can predict critical flow-field structures within the blade passage, providing industrial fan designers with the necessary insight to develop a design.

An industrial fan designer would classically develop a design to delay the onset of stall or improve fan efficiency at a desired operating point. However, insight into, for example, the nature of blade tip leakage flow features can facilitate the development of blade tip features that minimise the tip leakage vortex's intensity while maintaining its vorticity above a critical threshold value such that it does not burst. Thus, an industrial fan designer is able to effectively develop blade tip treatments that minimise fan acoustic emissions. As such, insight into an axial or centrifugal fan's blade-to-blade flow-field facilitates the achievement of a broad range of development goals.

10.3. Future Trends. Industrial fan designers have successfully integrated commercial computational fluid dynamic codes into the design process. The cost of the necessary computer hardware to run these codes has become progressively more affordable. Despite the reduced computer hardware costs, commercial computational fluid dynamic codes remain out of reach of many industrial fan designers working for smaller fan companies. This is because the cost of a licence for a commercial code remains high. The code author's policy of charging a licence fee for each processor results in a commercial code licence for a multiprocessor computer being typically an order of magnitude larger than the hardware's cost.

Industrial fan designers are increasingly working with the codes OpenFOAM and CODE_SATURNE. These codes are licence-free and can run on clusters of computers. This offers industrial fan designers the possibility of networking office computers. Computers that the engineering department uses during the day with design software may be used at night as part of a cluster to run open-source computational fluid dynamics codes. At the time of writing OpenFOAM and CODE_SATURNE have user interfaces that are less user friendly than commercial codes. Despite this caveat, 
the open-source movement generally has made dramatic progress over the last five years. Therefore, it is likely that open-source computational fluid dynamic codes will become dominant within the industrial fan community, as that community struggles to develop more efficient products in response to current and planned future legislation mandating minimum fan and motor efficiency.

\section{Nomenclature}

\section{Latin}

$U:$
$x, y, z:$
$i, j, k:$
$K:$
$y_{n}$
CFL $=U \times \Delta t / \Delta x:$

$\overline{u_{i}^{\prime} u_{j}^{\prime}}:$
$c_{\mu}, c_{1}, \ldots, c_{7}:$
$v^{2}:$
$F:$
$S_{i j}:$
$Q=0.5 \times\left(\Omega_{i j} \Omega_{i j}-S_{i j} S_{i j}\right):$
$y^{+}=y_{n} \times u_{\tau} / v:$
$\operatorname{Re}=U_{\mathrm{ref}} \times L_{\mathrm{ref}} / v:$
$\operatorname{Re}_{\tau}:$
$P_{\varphi}:$
$u_{\tau}=\tau_{w}^{0.5}:$
$T:$

Velocity $[\mathrm{m} / \mathrm{s}]$

Cartesian coordinates [m]

Indices [-]

Turbulent kinetic energy $\left[\mathrm{m}^{2} /\right.$ $\left.\mathrm{s}^{2}\right]$

Distance from the wall [m] [-]

Courant-Friedrichs-Lewy number, usually referred to as CFL or Courant number [-]

Reynolds stresses $\left[\mathrm{m}^{2} / \mathrm{s}^{2}\right]$

Numerical coefficients [-]

Normal-to-the-wall component of the Reynolds stresses $\left[\mathrm{m}^{2} / \mathrm{s}^{2}\right]$ Elliptic relaxation function [1/ s] Rate of strain tensor (symmetric component of the velocity gradient) $[1 / \mathrm{s}]$

$Q$ criterion for vortical structures deduction $\left[1 / \mathrm{s}^{2}\right]$ Normalised wall distance [-] Reynolds number based on reference velocity and length Reynolds number based on friction velocity

Production of $\varphi$ Friction velocity $[\mathrm{m} / \mathrm{s}]$ Time [s].

\section{Greek}

$\begin{array}{ll}\varepsilon: & \text { Dissipation rate of turbulent } \\ & \text { kinetic energy }\left[\mathrm{m}^{2} / \mathrm{s}^{3}\right] \\ \omega: & \text { Turbulence frequency }[1 / \mathrm{s}] \\ \delta_{i j}: & \text { Kronecker delta } \\ v: & \text { Kinematic viscosity }\left[\mathrm{m}^{2} / \mathrm{s}\right] \\ v_{t}: & \text { Turbulent kinematic viscosity } \\ \kappa=0.41: & \text { or eddy viscosity }\left[\mathrm{m}^{2} / \mathrm{s}\right] \\ \varphi: & \text { von Karman constant }[-] \\ \zeta=v^{2} / k: & \text { Generic quantity } \\ \Omega_{i j}: & \text { Reta [-] } \\ & \text { Rate of rotation tensor } \\ & \text { (antisymmetric component of } \\ & \text { the velocity gradient) }[1 / \mathrm{s}]\end{array}$

$\varepsilon_{\varphi}:$ Dissipation of $\varphi$

$\Delta_{1}$ : Distance from the wall of the first cell centre $[\mathrm{m}]$

$\tau_{w}:$ Wall shear stress $\left[\mathrm{m}^{2} / \mathrm{s}^{2}\right]$.

\section{Acronyms}

BEM: Boundary element method

CFD: Computational fluid dynamics

DES: Detached eddy simulations

DNS: Direct numerical simulation

HPC: High performance computing

LES: $\quad$ Large eddy simulations[-]

RSM: $\quad$ Reynolds stress models (same as SMC)

SAS: $\quad$ Scale adaptive simulations

SMC: $\quad$ Second moment closure (same as RSM)

URANS: Unsteady Reynolds-averaged Navier-Stokes

SST: $\quad$ Shear stress transport.

\section{Conflict of Interests}

The authors declare that there is no conflict of interests regarding the publication of this paper.

\section{References}

[1] B. Lakshminarayana, Fluid Dynamics and Heat Transfer of Turbomachinery, Wiley-Interscience, New York, NY, USA, 1996.

[2] N. A. Cumpsty, Compressor Aerodynamics, Krieger Publishing Company, Malabar, Fla, USA, 2004.

[3] C. Tropea, A. L. Yarin, and J. F. Foss, Eds., Springer Handbook of Experimental Fluid Mechanics, Springer, Paris, France, 2007.

[4] W. K. Blake, Mechanics of Flow-Induced Sound and Vibration, Volume 1: General Concepts and Elementary Sources, Academic Press, Orlando, Fla, USA, 1986.

[5] W. K. Blake, Mechanics of Flow-Induced Sound and Vibration, Volume 2: Complex Flow-Structure Interactions, Academic Press, Orlando, Fla, USA, 1986.

[6] L. Huang and J. Wang, "Acoustic analysis of a computer cooling fan," Journal of the Acoustical Society of America, vol. 118, no. 4, pp. 2190-2200, 2005.

[7] S. Bianchi, A. Corsini, and A. G. Sheard, "Demonstration of a stall detection system for induced draft fans," Proceedings of the Institution of Mechanical Engineers A, vol. 227, pp. 272-284, 2013.

[8] Commission Regulation (EU), No. 327/2011, Official Journal of the European Union, June 2011, http://www.amca.org/UserFiles/file/COMMISSION\%20REGULATION\%20\%28EU\%29\% 20No\%20327-2011.pdf.

[9] A. Hauer and J. Brooks, "Fan motor efficiency grades in the European market," AMCA Inmotion, no. 2, pp. 14-20, 2012.

[10] U.S. Department of Energy, Energy Conservation Standards Rulemaking Framework for Commercial and Industrial Fans and Blowers, U.S. Department of Energy, 2013.

[11] S. L. Gho, "AMCA grows in Asia," AMCA Inmotion, no. 3, 2013.

[12] J. H. Horlock and J. D. Denton, "A review of some early design practice using computational fluid dynamics and a current perspective," Journal of Turbomachinery, vol. 127, no. 1, pp. 513, 2005. 
[13] P. K. Kundu and I. M. Cohen, Fluid Mechanics, Elsevier Academic Press, Oxford, UK, 4th edition, 2008.

[14] P. A. Davidson, Turbulence, An Introduction for Scientists and Engineers, Oxford University Press, Oxford, UK, 2004.

[15] K. Hanjalić, S. Kenjeres, M. J. Tummers, and H. J. J. Jonker, Analysis and Modelling of Physical Transport Phenomena, VSSD, Delft, The Netherlands, 2008.

[16] P. A. Durbin and B. A. Pettersson Reif, Statistical Theory and Modelling for Turbulent Flows, John Wiley \& Sons, Chichester, UK, 2001.

[17] C. Hirsch, Numerical Computation of Internal and External Flows, Butterworth-Heinemann, Oxford, UK, 2007.

[18] S. B. Pope, Turbulent Flows, Cambridge University Press, Cambridge, UK, 2000.

[19] P. R. Spalart and S. R. Allmaras, "A one-equation turbulence model for aerodynamic flows," in Proceedings of the 30th Aerospace Sciences Meeting and Exhibit, AIAA Paper 92-0439, Reno, Nev, USA, January 1992.

[20] G. Rábai and J. Vad, "Validation of a computational fluid dynamics method to be applied to linear cascades of twistedswept blades," Periodica Polytechnica, Mechanical Engineering, vol. 49, no. 2, pp. 163-180, 2005.

[21] S. Šarić, B. Kniesner, A. Mehdizadeh, S. Jakirlić, K. Hanjalić, and C. Tropea, "Comparative assessment of hybrid LES/RANS models in turbulent flows separating from smooth surfaces," in Advances in Hybrid RANS-LES Modelling, Notes on Numerical Fluid Mechanics and Multidisciplinary Design, S. H. Peng and W. Haase, Eds., vol. 97, pp. 142-151, 2008.

[22] W. P. Jones and B. E. Launder, "The prediction of laminarization with a two-equation model of turbulence," International Journal of Heat and Mass Transfer, vol. 15, no. 2, pp. 301-314, 1972.

[23] D. C. Wilcox, "Reassessment of the scale-determining equation for advanced turbulence models," AIAA Journal, vol. 26, no. 11, pp. 1299-1310, 1988.

[24] D. C. Wilcox, Turbulence Modelling for CFD, DCW Industries, La Canada, Canada, 1993.

[25] F. R. Menter, "Zonal two-equation k-w turbulence model for aerodynamic flows," in Proceedings of the 24th Fluid Dynamics Conference, AIAA Paper 1993-2906, Orlando, Fla, USA, July 1993.

[26] F. R. Menter, "Two-equation eddy-viscosity turbulence models for engineering applications," AIAA Journal, vol. 32, no. 8, pp. 1598-1605, 1994.

[27] M. Pinelli, C. Ferrari, A. Suman, M. Morini, and M. Rossini, "Fluid dynamic design and optimization of a double entry fan driven by tractor power take off for mist sprayer applications," in Proceedings of the Fan 2012 Conference, Senlis, France, April 2012.

[28] A. Corsini and F. Rispoli, "Flow analyses in a high-pressure axial ventilation fan with a non-linear eddy-viscosity closure," International Journal of Heat and Fluid Flow, vol. 17, pp. 108-155, 2005.

[29] A. G. Sheard, A. Corsini, S. Minotti, and F. Sciulli, "The role of computational methods in the development of an aero-acoustic design methodology: application in a family of large industrial fans," in Proceedings of the 14th International Conference on Modelling Fluid Flow Technologies, pp. 71-79, Budapest, Hungary, September 2009.

[30] A. Corsini and F. Rispoli, "Using sweep to extend the stall-free operational range in axial fan rotors," Proceedings of the Institution of Mechanical Engineers A, vol. 218, no. 3, pp. 129-139, 2004.
[31] A. Corsini and A. G. Sheard, "Tip end-plate concept based on leakage vortex rotation number control," Journal of Computational and Applied Mechanics, vol. 8, pp. 21-37, 2007.

[32] A. Corsini, F. Rispoli, and A. G. Sheard, "Development of improved blade tip endplate concepts for low-noise operation in industrial fans," Proceedings of the Institution of Mechanical Engineers A, vol. 221, no. 5, pp. 669-681, 2007.

[33] A. Corsini, F. Rispoli, and A. G. Sheard, "Aerodynamic performance of blade tip end-plates designed for low-noise operation in Axial flow fans," Journal of Fluids Engineering, vol. 131, no. 8, Article ID 0811011, 13 pages, 2009.

[34] A. Corsini, F. Rispoli, and A. G. Sheard, "Shaping of tip endplate to control leakage vortex swirl in axial flow fans," Journal of Turbomachinery, vol. 132, no. 3, Article ID 031005, 9 pages, 2010.

[35] J. Vad and C. Horváth, "Study on the effects of axial clearance size on the operation of an axial flow electric motor cooling fan," in Proceedings of the 10th European Turbomachinery Conference, Lappeenranta, Finland, April 2013.

[36] A. Corsini, G. Delibra, and A. G. Sheard, "Leading edge bumps in reversible axial fans," in Proceedings of 58th American Society of Mechanical Engineers Gas Turbine and Aeroengine Congress, Paper No. GT2013-94853, San Antonio, Tex, USA, June 2013.

[37] F. S. Lien, W. L. Chen, and M. A. Leschziner, "Low-Reynoldsnumber eddy-viscosity modelling based on non-linear stressstrain/vorticity relations," in Proceedings of the 3rd Symposium on Engineering Turbulence Modelling and Measurements, Crete, Greece, May 1996.

[38] S. Lee, S. Heo, and C. Cheong, "Prediction and reduction of internal blade-passing frequency noise of the centrifugal fan in a refrigerator," International Journal of Refrigeration, vol. 33, no. 6, pp. 1129-1141, 2010.

[39] S. J. van der Spuy, T. W. von Backström, and D. G. Kröger, "An evaluation of simplified methods to model the performance of axial flow fan arrays," $R$ \& $D$ Journal of the South African Institution of Mechanical Engineering, vol. 26, pp. 12-20, 2010.

[40] A. Corsini, F. Rispoli, A. G. Sheard, and P. Venturini, "Numerical simulation of coal-fly ash erosion in an induced draft fan," Journal of Fluids Engineering, vol. 135, Article ID 081303, 12 pages, 2013.

[41] D. Borello, A. Corsini, F. Rispoli, and A. G. Sheard, "Large eddy simulation of a tunnel ventilation fan," Journal of Fluids Engineering, vol. 135, Article ID 071102, 9 pages, 2013.

[42] M. Popovac and K. Hanjalić, "Compound wall treatment for RANS computation of complex turbulent flows and heat transfer," Flow, Turbulence and Combustion, vol. 78, no. 2, pp. 177202, 2007.

[43] T. J. Craft, B. E. Launder, and K. Suga, "Development and application of a cubic eddy-viscosity model of turbulence," International Journal of Heat and Fluid Flow, vol. 17, no. 2, pp. 108-115, 1996.

[44] W. L. Chen, F. S. Lien, and M. A. Leschziner, "Computational prediction of flow around highly loaded compressor-cascade blades with non-linear eddy-viscosity models," International Journal of Heat and Fluid Flow, vol. 19, no. 4, pp. 307-319, 1998.

[45] K. Hanjalić, M. Popovac, and M. Hadžiabdić, "A robust nearwall elliptic-relaxation eddy-viscosity turbulence model for CFD," International Journal of Heat and Fluid Flow, vol. 25, no. 6, pp. 1047-1051, 2004.

[46] P. A. Durbin, "Separated flow computations with the k-epsilonv-squared model," AIAA Journal, vol. 33, pp. 659-664, 1995. 
[47] B. E. Launder and N. D. Sandham, Eds., Closure Strategies for Turbulent and Transitional Flows, Cambridge University Press, Cambridge, UK, 2002.

[48] K. Hanjalić and B. Launder, Modelling Turbulence in Engineering and the Environment: Second-Moment Routes to Closure, Cambridge University Press, Cambridge, UK, 2011.

[49] D. Borello, K. Hanjalić, and F. Rispoli, "Prediction of cascade flows with innovative second-moment closures," Journal of Fluids Engineering, vol. 127, no. 6, pp. 1059-1070, 2005.

[50] K. Hanjalić and S. Jakirlić, "Contribution towards the secondmoment closure modelling of separating turbulent flows," Computer and Fluids, vol. 27, pp. 137-156, 1998.

[51] K. Hanjalić and S. Jakirlić, "A model of stress dissipation in second-moment closures," Applied Scientific Research, vol. 51, pp. 513-518, 1993.

[52] P. A. Durbin, "A Reynolds stress model for near-wall turbulence," Journal of Fluid Mechanics, vol. 249, pp. 465-498, 1993.

[53] D. Borello, K. Hanjalić, and F. Rispoli, "Computation of tipleakage flow in a linear compressor cascade with a secondmoment turbulence closure," International Journal of Heat and Fluid Flow, vol. 28, no. 4, pp. 587-601, 2007.

[54] T. Kobayashi and M. Yoda, "Modified k-epsilon model for turbulent swirling flow in a straight pipe," JSME International Journal, vol. 30, no. 259, pp. 66-71, 1987.

[55] G. F. Sander and D. G. Lilley, "The performance of an annular vane swirler," in Proceedings of the AIAA/SAE/ASME 19th Joint Propulsion Conference, Paper No. AIAA-83-1326, Seattle, Wash, USA, 1983.

[56] C. G. Speziale, S. Sarkar, and T. B. Gatski, "Modelling the pressure-strain correlation of turbulence. An invariant dynamical systems approach," Journal of Fluid Mechanics, vol. 227, pp. 245272, 1991.

[57] F. S. Lien and P. A. Durbin, "Non linear k- $\varepsilon$-v2 modelling with application to high lift," in Proceedings of the CTR Summer Program, Stanford University, 1996.

[58] R. B. Langtry and F. R. Menter, "Transition modeling for general CFD applications in aeronautics," in Proceedings of the 43rd AIAA Aerospace Sciences Meeting and Exhibit, AIAA Paper No. 2005-522, pp. 15513-15526, Reno, Nev, USA, January 2005.

[59] F. R. Menter, R. B. Langtry, S. Völker, and P. G. Huang, "Transition modelling for general purpose CFD codes," Flow, Turbulence and Combustion, vol. 77, pp. 277-303, 2006.

[60] F. R. Menter, R. Langtry, and S. Volker, "Transition modelling for general purpose CFD codes," Flow, Turbulence and Combustion, vol. 77, pp. 277-303, 2006.

[61] W. Piotrowski, W. Elsner, and S. Drobniak, "Transition prediction on turbine blade profile with intermittency transport equation," in Proceedings of the 53rd American Society of Mechanical Engineers Gas Turbine and Aeroengine Congress, Paper No. GT2008-50796, Berlin, Germany, June 2008.

[62] Y. Yang, A. Lucius, and G. Brenner, "3D unsteady CFD simulation of the pressure fluctuation in a centrifugal fan," in Proceedings of the Fan 2012 Conference, Senlis, France, April 2012.

[63] J. D. Denton and W. N. Dawes, "Computational fluid dynamics for turbomachinery design," Proceedings of the Institution of Mechanical Engineers C, vol. 213, no. 2, pp. 107-124, 1999.

[64] J. D. Denton, Extension of the Finite Volume Time Marching Method to Three Dimensions, VKI Lecture Series 1979-7, VKI, Rhode-St-Genèse, Belgium, 1979.

[65] M. Vanella, U. Piomelli, and E. Balaras, "Effect of grid discontinuities on large-eddy simulation statistics and flow fields," Journal of Turbulence, vol. 9, article 32, 2008.
[66] G. D. Thiart and T. W. von Backström, "Numerical simulation of the flow field near an axial flow fan operating under distorted inflow conditions," Journal of Wind Engineering and Industrial Aerodynamics, vol. 45, no. 2, pp. 189-214, 1993.

[67] J. M. F. Oro, K. M. A. Díaz, C. S. Morros, and E. B. Marigorta, "Unsteady flow and wake transport in a low-speed axial fan with inlet guide vanes," Journal of Fluids Engineering, vol. 129, no. 8, pp. 1015-1029, 2007.

[68] I. A. Brailko, V. I. Mileshin, M. A. Nyukhtikov, and S. V. Pankov, "Computational and experimental investigation of unsteady and acoustic characteristics of counter-rotating fans," in Proceedings of the ASME Heat Transfer/Fluids Engineering Summer Conference, Parts A and B, vol. 2 of Paper No. HT-FED200456435, pp. 871-879, Charlotte, NC, USA, July 2004.

[69] D. Wolfram and T. H. Carolus, "Experimental and numerical investigation of the unsteady flow field and tone generation in an isolated centrifugal fan impeller," Journal of Sound and Vibration, vol. 329, no. 21, pp. 4380-4397, 2010.

[70] P. N. Son, J. Kim, and E. Y. Ahn, "Effects of bell mouth geometries on the flow rate of centrifugal blowers," Journal of Mechanical Science and Technology, vol. 25, no. 9, pp. 2267-2276, 2011.

[71] D. Angeli, "HPC enabling of OpenFOAM for CFD applications," in Proceedings of the OpenFOAM @ Mimesis and Numerical Simulation of the Mont Blanc Tunnel Workshop, CINECA, Bologna, Italy, November 2012.

[72] A. Betz, Introduction to the Theory of Flow Machines, Pergamon Press, Oxford, UK, 1966, (D. G. Randall, Translation).

[73] C. J. Meyer and D. G. Kröger, "Numerical simulation of the flow field in the vicinity of an axial flow fan," International Journal for Numerical Methods in Fluids, vol. 36, no. 8, pp. 947-969, 2001.

[74] J. N. Sørensen and W. Z. Shen, "Numerical modelling of wind turbine wakes," Journal of Fluids Engineering, vol. 124, pp. 393399, 2002.

[75] P. Venturini, Modelling of particle-wall deposition in two-phase gas-solid flow [Ph.D. thesis], Sapienza Università di Roma, Rome, Italy, 2010.

[76] L. L. Baxter, Turbulent transport of particles [Ph.D. thesis], Brigham Young University, Provo, Utah, USA, 1989.

[77] W. Tabakoff, R. Kotwal, and A. Hamed, "Erosion study of different materials affected by coal ash particles," Wear, vol. 52, pp. 161-173, 1979.

[78] A. Ghenaiet, "Numerical study of sand ingestion through a ventilating system," in Proceedings of the World Congress on Engineering, vol. 2, London, UK, July 2009.

[79] L. He and J. D. Denton, "Three-dimensional time-marching inviscid and viscous solutions for unsteady flows around vibrating blades," Journal of Turbomachinery, vol. 116, no. 3, pp. 469476, 1994.

[80] J. G. Marshall and M. Imregun, "A review of aeroelasticity methods with emphasis on turbomachinery applications," Journal of Fluids and Structures, vol. 10, no. 3, pp. 237-267, 1996.

[81] E. Envia, A. G. Wilson, and D. L. Huff, "Fan noise: a challenge to CAA," International Journal of Computational Fluid Dynamics, vol. 18, no. 6, pp. 471-480, 2004.

[82] F. R. Menter and Y. Egorov, "A scale-adaptive simulation model using two-equation models," in Proceedings of the 43rd AIAA Aerospace Sciences Meeting and Exhibit, AIAA Paper No. 20051095, pp. 271-283, Reno, Nev, USA, January 2005.

[83] F. R. Menter and Y. Egorov, "The scale-adaptive simulation method for unsteady turbulent flow predictions, part 1: theory and model description," Flow, Turbulence and Combustion, vol. 85, no. 1, pp. 113-138, 2010. 
[84] F. R. Menter and Y. Egorov, "The scale-adaptive simulation method for unsteady turbulent flow predictions, part 2: application to complex flows," Flow, Turbulence and Combustion, vol. 85, no. 1, pp. 139-165, 2010.

[85] D. Borello, G. Delibra, K. Hanjalić, and F. Rispoli, "Largeeddy simulations of tip leakage and secondary flows in an axial compressor cascade using a near-wall turbulence model," Proceedings of the Institution of Mechanical Engineers A, vol. 223, no. 6, pp. 645-655, 2009.

[86] L. Davidson, "Large eddy simulation: a dynamic one-equation subgrid model for three-dimensional recirculating flow," in Proceedings of the 11th International Symposium on Turbulent Shear Flow, vol. 3, pp. 26.1-26.6, Grenoble, France, 1997.

[87] H. Jasak, "OpenFOAM: a year in review," in Proceedings of the 5th OpenFOAM Workshop, Gothenburg, Sweden, June 2010.

[88] A. Frederic, N. Mehitoua, and S. Marc, "Code_Saturne: a .finite volume code for the computation of turbulent incompressible flows-industrial applications," IJFV International Journal on Finite Volumes, vol. 1, pp. 1-62, 2004.

[89] M. S. Eldred, A. A. Giunta, B. G. van Bloemen Waanders, S. F. Wojtkiewicz, W. E. Hart, and M. P. Alleva, "DAKOTA, a multilevel parallel object-oriented framework for design optimization, parameter estimation, uncertainty quantification, and sensitivity analysis," Sand Report SAND2001-3796, Sandia National Laboratories, Livermore, Calif, USA, 2002.

[90] I. Spisso, "HPC Enabling of OpenFOAM for CFD Applications," in Parametric and Optimization Study: OpenFOAM and Dakota, Proceedings of Workshop, CINECA, Bologna, Italy, November 2012. 

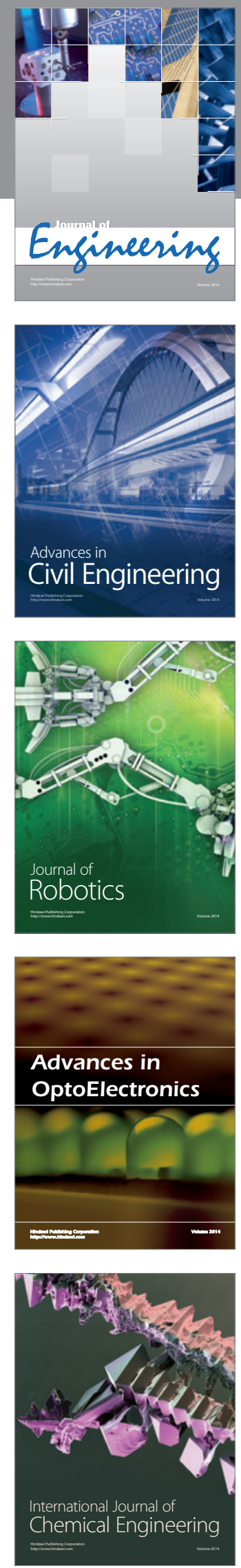

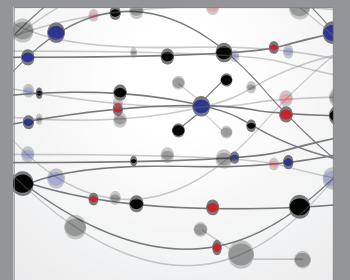

The Scientific World Journal
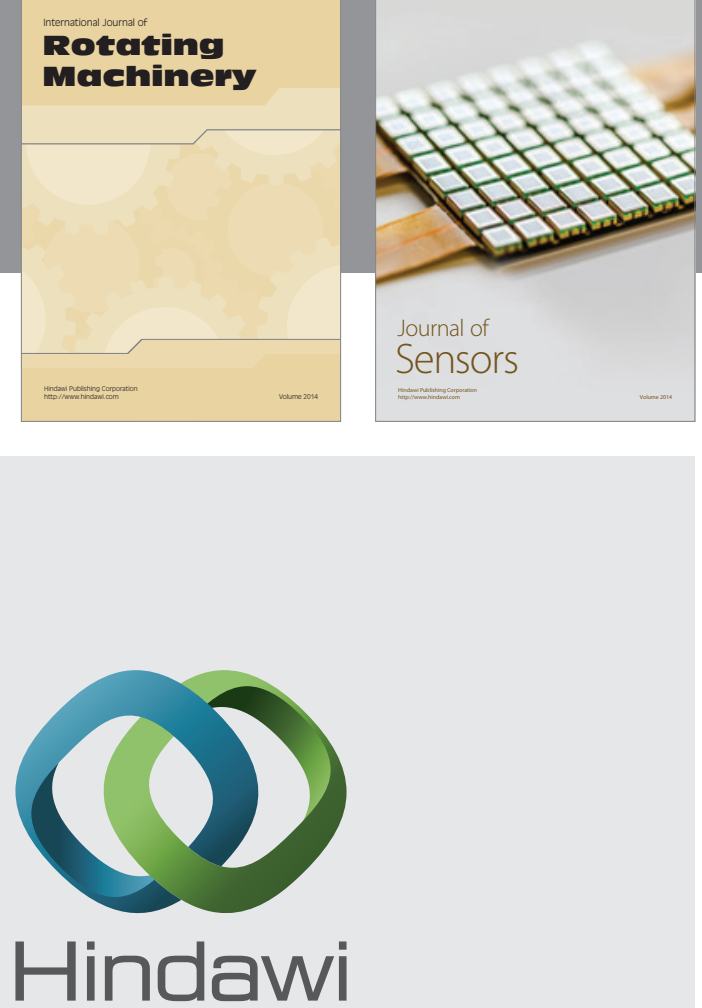

Submit your manuscripts at http://www.hindawi.com
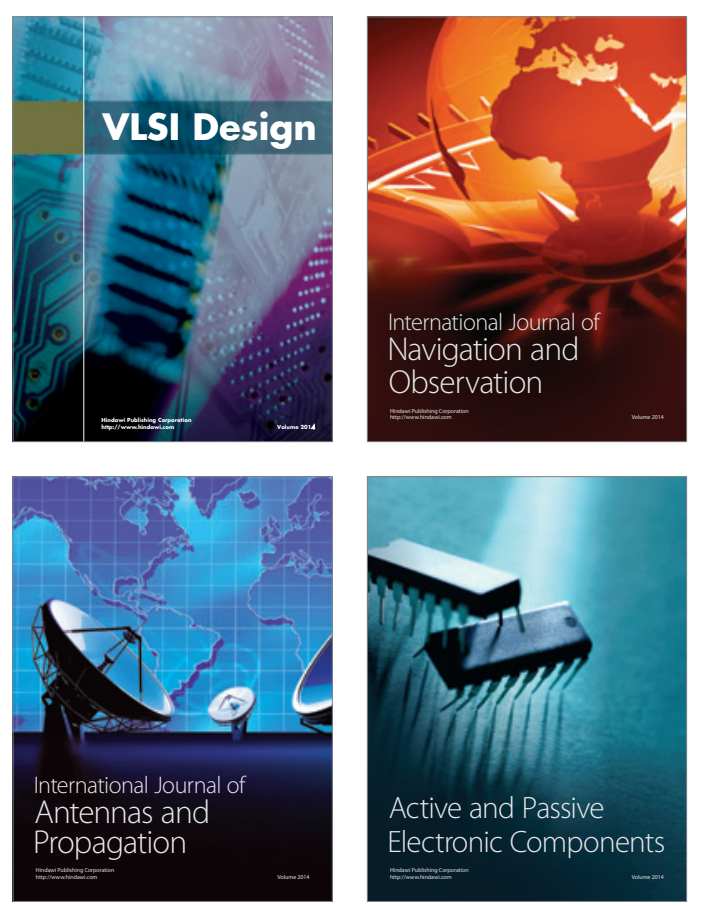
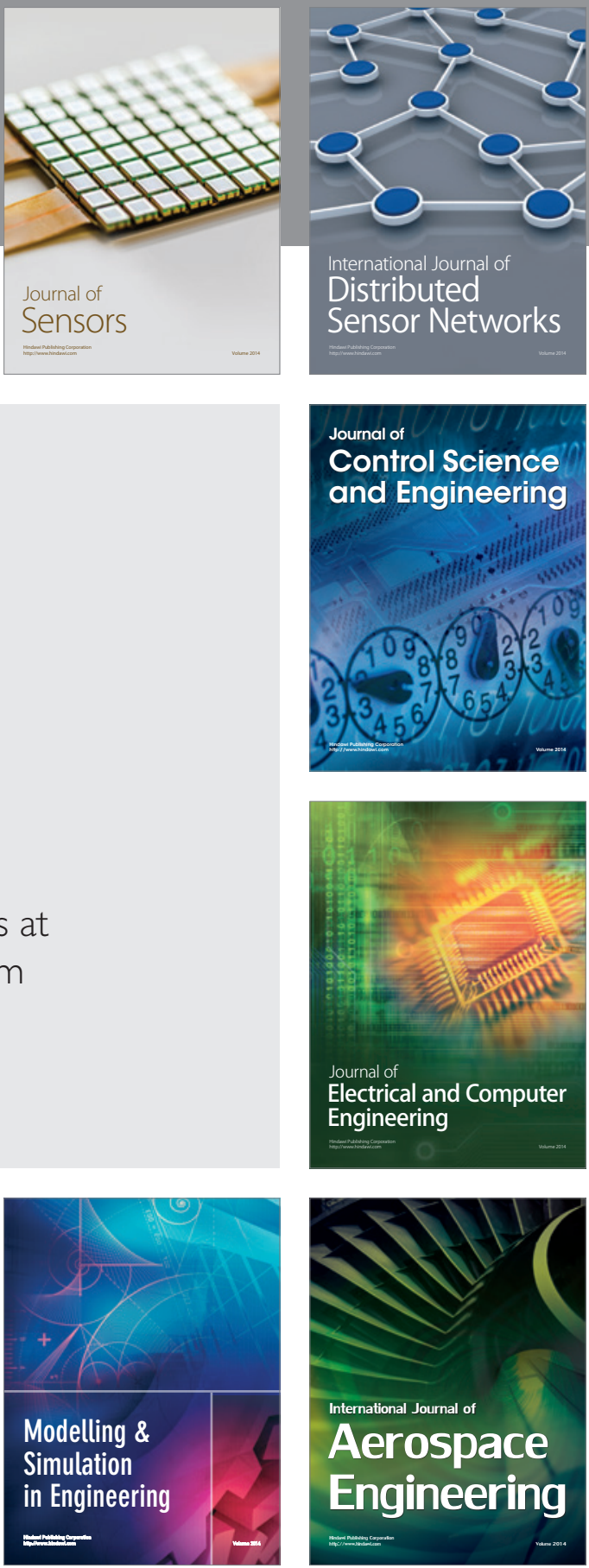

Journal of

Control Science

and Engineering
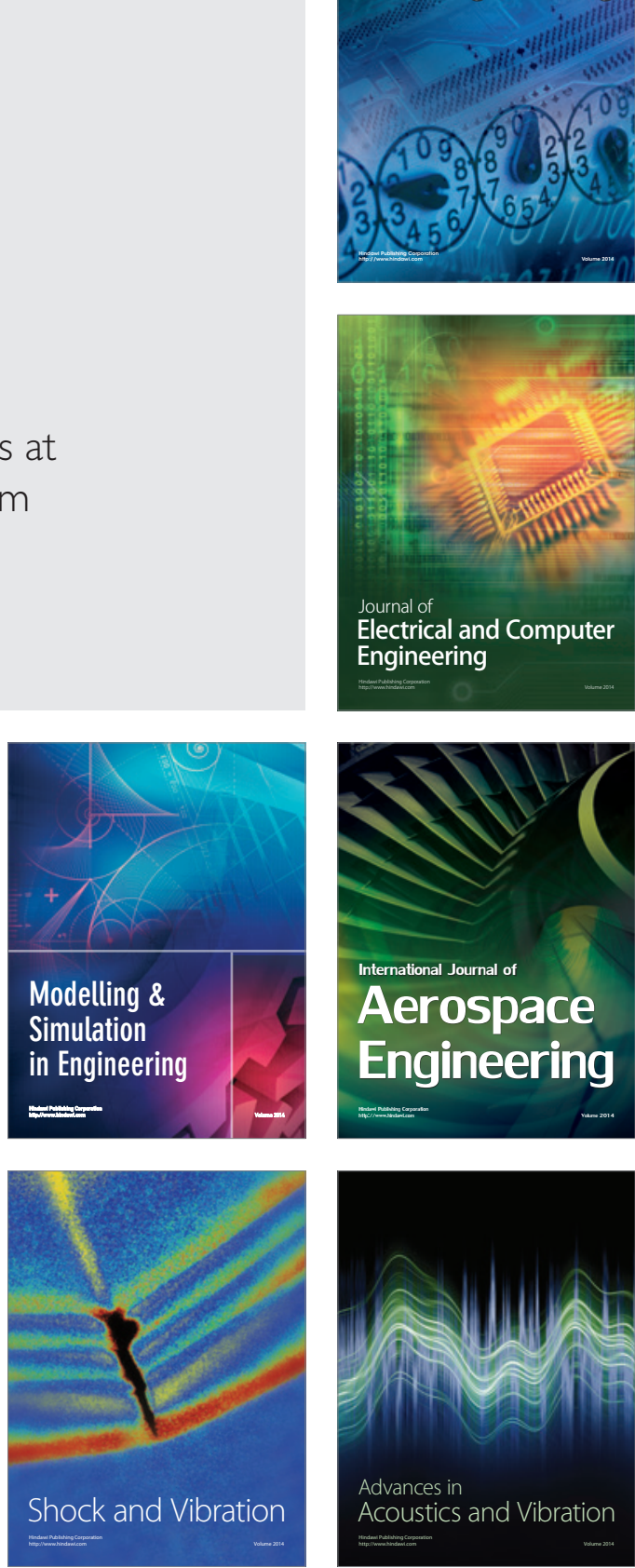